Supporting information for

Computational Modeling of Ansa-Zirconocene Amide Complexes

\author{
Adam R. Dunn, ${ }^{a}$ Lucas E. Sweet, ${ }^{a}$ Dawn C. Wiser, ${ }^{a} *$ Matthew LoCoco ${ }^{b}$ \\ and Richard F. Jordan ${ }^{b, *}$
}

${ }^{a}$ Department of Chemistry, Lake Forest College, 555 N. Sheridan Road, Lake Forest, IL 60045 and ${ }^{b}$ Department of Chemistry, The University of Chicago, 5735 South Ellis Avenue, Chicago, IL 60637

Listing of cartesian coordinates, absolute energies (in hartrees), and number of imaginary frequencies for all structures reported in Tables 1-11. 


\section{rac-(MBSBI)Zr $\left\{\mathrm{PhN}\left(\mathrm{CH}_{2}\right)_{3} \mathrm{NPh}\right\}(3)$ boat (rac-3) \\ absolute energy: $\mathbf{- 1 8 9 9 . 8 9 2 0 4 5 3 2}$ hartrees imaginary frequencies: 0}

\begin{tabular}{|c|c|c|c|c|c|}
\hline \multirow{2}{*}{$\begin{array}{l}\text { Center } \\
\text { Number }\end{array}$} & \multirow{2}{*}{$\begin{array}{l}\text { Atomic } \\
\text { Number }\end{array}$} & \multirow{2}{*}{\multicolumn{2}{|c|}{$\begin{array}{l}\text { Atomic } \\
\text { Type }\end{array}$}} & \multicolumn{2}{|c|}{ Coordinates (Angstrom } \\
\hline & & & & $\mathrm{X}$ & $\mathrm{Z}$ \\
\hline 1 & 40 & 0 & -.094845 & -.144096 & .161968 \\
\hline 2 & 7 & 0 & 1.121911 & .378133 & 1.766017 \\
\hline 3 & 7 & 0 & -1.207040 & 1.576980 & .362360 \\
\hline 4 & 6 & 0 & -1.244103 & -2.531201 & -.544051 \\
\hline 5 & 6 & 0 & -.620690 & -2.698317 & .750887 \\
\hline 6 & 6 & 0 & -1.392623 & -1.965084 & 1.727479 \\
\hline 7 & 6 & 0 & -2.533863 & -1.388872 & 1.066741 \\
\hline 8 & 6 & 0 & -2.426326 & -1.713830 & -.351141 \\
\hline 9 & 6 & 0 & .603449 & -1.153933 & -2.123786 \\
\hline 10 & 6 & 0 & $.058 \varepsilon$ & .143953 & -2.466355 \\
\hline 11 & 6 & 0 & .947456 & 1.157863 & -1.972812 \\
\hline 12 & 6 & 0 & 2.114542 & .518420 & -1.412945 \\
\hline 13 & 6 & 0 & 1.902097 & -.917665 & -1.495285 \\
\hline 14 & 6 & 0 & -1.71 & 2.030589 & 1.730491 \\
\hline 15 & 6 & 0 & .596960 & 1.400320 & 2.777204 \\
\hline 16 & 6 & 0 & 2.503850 & .024060 & 2.131230 \\
\hline 17 & 6 & 0 & -1.6 & 2.593874 & -.619975 \\
\hline 18 & 1 & 0 & -1.277406 & -2.090391 & 2.808597 \\
\hline 19 & 6 & 0 & .411 & -3.768487 & 1.133696 \\
\hline 20 & 6 & 0 & -3.519884 & -1.371456 & -1.247426 \\
\hline 21 & 6 & 0 & -1.10 & .434761 & -3.418490 \\
\hline 22 & 6 & 0 & 3.006462 & -1.821321 & -1.196130 \\
\hline 23 & 6 & 0 & 3.430795 & 1.066116 & -1.066485 \\
\hline 24 & 6 & 0 & -3.761399 & -.780370 & 1.599908 \\
\hline 25 & 1 & 0 & .859689 & 2.222188 & -2.209854 \\
\hline 26 & 6 & 0 & -.950535 & 1.392948 & 2.928296 \\
\hline 27 & 14 & 0 & -.350800 & -2.843417 & -2.238401 \\
\hline 28 & 6 & 0 & 3.464807 & 1.034125 & 2.430473 \\
\hline 29 & 6 & 0 & 2.891089 & -1.334180 & 2.259389 \\
\hline 30 & 6 & 0 & -2.864964 & 2.411320 & -1.338022 \\
\hline 31 & 6 & 0 & -.920893 & 3.795565 & -.792931 \\
\hline 32 & 1 & 0 & -1.583595 & 3.131688 & 1.772168 \\
\hline 33 & 1 & 0 & -2.794586 & 1.806216 & 1.820360 \\
\hline 34 & 1 & 0 & 1.038985 & 1.152163 & 3.763528 \\
\hline 35 & 1 & 0 & .935567 & 2.417228 & 2.493751 \\
\hline 36 & 6 & 0 & -1.538779 & -3.020363 & -3.815118 \\
\hline 37 & 6 & 0 & .807573 & -4.449262 & -2.296833 \\
\hline
\end{tabular}




\begin{tabular}{|c|c|c|c|c|c|}
\hline 38 & 6 & 0 & -4.813206 & -.461011 & .666387 \\
\hline 39 & 6 & 0 & -4.650081 & -.765895 & -.754611 \\
\hline 40 & 1 & 0 & -3.457679 & -1.650926 & -2.306422 \\
\hline 41 & 6 & 0 & -3.983773 & -.556603 & 2.994182 \\
\hline 42 & 6 & 0 & 4.246795 & -1.306959 & -.906734 \\
\hline 43 & 6 & 0 & 4.503202 & .130936 & -.840632 \\
\hline 44 & 1 & 0 & 2.855433 & -2.904404 & -1.278928 \\
\hline 45 & 6 & 0 & 3.704228 & 2.467633 & -1.020133 \\
\hline 46 & 1 & 0 & .005317 & -4.768293 & .897332 \\
\hline 47 & 1 & 0 & 1.368539 & -3.659833 & .605317 \\
\hline 48 & 1 & 0 & .607541 & -3.727270 & 2.215538 \\
\hline 49 & 1 & 0 & -.850012 & .069826 & -4.428929 \\
\hline 50 & 1 & 0 & -2.041306 & -.049332 & -3.105619 \\
\hline 51 & 1 & 0 & -1.284749 & 1.517958 & -3.472671 \\
\hline 52 & 1 & 0 & 3.191170 & 2.091726 & 2.330670 \\
\hline 53 & 6 & 0 & 4.761731 & .684398 & 2.839594 \\
\hline 54 & 1 & 0 & 2.145481 & -2.116487 & 2.078512 \\
\hline 55 & 6 & 0 & 4.196809 & -1.675756 & 2.660907 \\
\hline 56 & 1 & 0 & -3.441802 & 1.487406 & -1.187834 \\
\hline 57 & 6 & 0 & -3.313081 & 3.405497 & -2.231146 \\
\hline 58 & 1 & 0 & .009717 & 3.938050 & -.225663 \\
\hline 59 & 6 & 0 & -1.375276 & 4.782128 & -1.686467 \\
\hline 60 & 1 & 0 & -1.209792 & 1.974256 & 3.830828 \\
\hline 61 & 1 & 0 & -1.290300 & .359482 & 3.096347 \\
\hline 62 & 6 & 0 & -6.024522 & .116477 & 1.163363 \\
\hline 63 & 1 & 0 & -5.489118 & -.533633 & -1.423872 \\
\hline 64 & 1 & 0 & 5.094595 & -1.982546 & -.729337 \\
\hline 65 & 6 & 0 & 5.813767 & .639605 & -.570485 \\
\hline 66 & 6 & 0 & 4.992492 & 2.932318 & -.753580 \\
\hline 67 & 1 & 0 & 2.884441 & 3.177439 & -1.193542 \\
\hline 68 & 6 & 0 & -5.179627 & .000495 & 3.449290 \\
\hline 69 & 1 & 0 & -3.198830 & -.828865 & 3.711582 \\
\hline 70 & 1 & 0 & -2.144648 & -2.127744 & -3.991067 \\
\hline 71 & 1 & 0 & -.930926 & -3.206022 & -4.708585 \\
\hline 72 & 1 & 0 & -2.209388 & -3.876216 & -3.673882 \\
\hline 73 & 1 & 0 & 1.477935 & -4.392412 & -3.162529 \\
\hline 74 & 1 & 0 & 1.411638 & -4.568626 & -1.393496 \\
\hline 75 & 1 & 0 & .179889 & -5.341255 & -2.409646 \\
\hline 76 & 1 & 0 & 5.488959 & 1.476183 & 3.064959 \\
\hline 77 & 6 & 0 & 5.136020 & -.670517 & 2.956782 \\
\hline 78 & 1 & 0 & 4.471656 & -2.734285 & 2.762856 \\
\hline 79 & 1 & 0 & -4.248176 & 3.254141 & -2.787271 \\
\hline 80 & 6 & 0 & -2.571242 & 4.589133 & -2.408746 \\
\hline 81 & 1 & 0 & -.795090 & 5.705199 & -1.820150 \\
\hline 82 & 6 & 0 & 6.054675 & 2.012506 & -.527579 \\
\hline 83 & 1 & 0 & 6.631747 & -.072912 & -.397171 \\
\hline
\end{tabular}




$\begin{array}{lllrrl}84 & 1 & 0 & 5.191387 & 4.011883 & -.718455 \\ 85 & 6 & 0 & -6.203989 & .349892 & 2.526746 \\ 86 & 1 & 0 & -6.820838 & .367543 & .449476 \\ 87 & 1 & 0 & -5.333435 & .168969 & 4.523479 \\ 88 & 1 & 0 & 6.149897 & -.937482 & 3.281621 \\ 89 & 1 & 0 & -2.923248 & 5.361132 & -3.105603 \\ 90 & 1 & 0 & 7.065158 & 2.389047 & -.320053 \\ 91 & 1 & 0 & -7.139969 & .792003 & 2.893012\end{array}$

\section{chair (rac-3) \\ absolute energy: $-\mathbf{1 8 9 9 . 8 9 5 1 1 2 2 9}$ hartrees imaginary frequencies: 0}

\begin{tabular}{|c|c|c|c|c|c|}
\hline \multirow{2}{*}{$\begin{array}{l}\text { Center } \\
\text { Number }\end{array}$} & \multirow{2}{*}{$\begin{array}{l}\text { Atomic } \\
\text { Number }\end{array}$} & \multirow{2}{*}{\multicolumn{2}{|c|}{$\begin{array}{r}\text { Atomic } \\
\text { Type }\end{array}$}} & \multicolumn{2}{|c|}{ Coordinates (Angstroms } \\
\hline & & & & $\mathrm{X}$ & $\mathrm{Z}$ \\
\hline 1 & 40 & 0 & .102327 & .160555 & .176244 \\
\hline 2 & 7 & 0 & -1.116246 & -.443041 & 1.733530 \\
\hline 3 & 7 & 0 & 1.197492 & -1.583118 & .147928 \\
\hline 4 & 6 & 0 & 1.313668 & 2.555005 & -.052875 \\
\hline 5 & 6 & 0 & .735446 & 2.465737 & 1.268249 \\
\hline 6 & 6 & 0 & 1.531220 & 1.552380 & 2.055475 \\
\hline 7 & 6 & 0 & 2.667477 & 1.145650 & 1.268286 \\
\hline 8 & 6 & 0 & 2.518520 & 1.733257 & -.051257 \\
\hline 9 & 6 & 0 & -.596996 & 1.571207 & -1.854771 \\
\hline 10 & 6 & 0 & -.062152 & .342872 & -2.418875 \\
\hline 11 & 6 & 0 & -.963669 & -.730721 & -2.119298 \\
\hline 12 & 6 & 0 & -2.127015 & -.184279 & -1.457167 \\
\hline 13 & 6 & 0 & -1.903803 & 1.244678 & -1.294342 \\
\hline 14 & 6 & 0 & 1.536084 & -2.258750 & 1.479448 \\
\hline 15 & 6 & 0 & -.417697 & -1.227749 & 2.852743 \\
\hline 16 & 6 & 0 & -2.557130 & -.352651 & 2.047659 \\
\hline 17 & 6 & 0 & 1.639087 & -2.449134 & -.965644 \\
\hline 18 & 1 & 0 & 1.452707 & 1.470025 & 3.144136 \\
\hline 19 & 6 & 0 & -.320285 & 3.393350 & 1.883197 \\
\hline 20 & 6 & 0 & 3.587993 & 1.586547 & -1.023208 \\
\hline 21 & 6 & 0 & 1.111624 & .213000 & -3.396217 \\
\hline 22 & 6 & 0 & -3.007366 & 2.097687 & -.869996 \\
\hline 23 & 6 & 0 & -3.457454 & -.766812 & -1.238681 \\
\hline 24 & 6 & 0 & 3.890241 & .424990 & 1.631853 \\
\hline 25 & 1 & 0 & -.889309 & -1.736717 & -2.543366 \\
\hline 26 & 6 & 0 & .290573 & -2.530309 & 2.373432 \\
\hline 27 & 1 & 0 & 2.032592 & -3.222266 & 1.251871 \\
\hline 28 & 1 & 0 & 2.258664 & -1.630907 & 2.035255 \\
\hline 29 & 1 & 0 & -1.183235 & -1.490045 & 3.608492 \\
\hline
\end{tabular}




$\begin{array}{lccccc}30 & 1 & 0 & .330055 & -.573759 & 3.341478 \\ 31 & 14 & 0 & .400338 & 3.227279 & -1.629191 \\ 32 & 6 & 0 & -3.350106 & -1.532280 & 2.127116 \\ 33 & 6 & 0 & -3.150783 & .893175 & 2.369985 \\ 34 & 6 & 0 & 2.808795 & -2.133175 & -1.702558 \\ 35 & 6 & 0 & .928237 & -3.640770 & -1.277138 \\ 36 & 1 & 0 & -.429025 & -3.164933 & 1.827601 \\ 37 & 1 & 0 & .628201 & -3.082110 & 3.269818 \\ 38 & 6 & 0 & 1.568423 & 3.703287 & -3.157734 \\ 39 & 6 & 0 & -.716034 & 4.834404 & -1.324386 \\ 40 & 6 & 0 & 4.919920 & .300439 & .629729 \\ 41 & 6 & 0 & 4.729901 & .896300 & -.690118 \\ 42 & 1 & 0 & 3.499790 & 2.072287 & -2.002896 \\ 43 & 6 & 0 & 4.124109 & -.112585 & 2.937102 \\ 44 & 6 & 0 & -4.257564 & 1.557038 & -.701086 \\ 45 & 6 & 0 & -4.527099 & .130876 & -.885726 \\ 46 & 1 & 0 & -2.844107 & 3.177021 & -.765967 \\ 47 & 6 & 0 & -3.743850 & -2.151616 & -1.439536 \\ 48 & 1 & 0 & .093629 & 4.414506 & 1.973572 \\ 49 & 1 & 0 & -1.239507 & 3.453184 & 1.284904 \\ 50 & 1 & 0 & -.586275 & 3.040364 & 2.890466 \\ 51 & 1 & 0 & .853860 & .724087 & -4.341243 \\ 52 & 1 & 0 & 2.036387 & .655471 & -3.005015 \\ 53 & 1 & 0 & 1.303459 & -.847063 & -3.612987 \\ 54 & 1 & 0 & -2.899046 & -2.501122 & 1.874410 \\ 55 & 6 & 0 & -4.696443 & -1.457878 & 2.521811 \\ 56 & 1 & 0 & -2.536011 & 1.801317 & 2.337637 \\ 57 & 6 & 0 & -4.503413 & .959023 & 2.760261 \\ 58 & 1 & 0 & 3.372353 & -1.224271 & -1.446853 \\ 59 & 6 & 0 & 3.243976 & -2.982457 & -2.740191 \\ 60 & 1 & 0 & .023822 & -3.888733 & -.704530 \\ 61 & 6 & 0 & 1.371627 & -4.483197 & -2.311600 \\ 62 & 6 & 0 & 6.131385 & -.384658 & .968597 \\ 63 & 1 & 0 & 5.551742 & .806591 & -1.412816 \\ 64 & 1 & 0 & -5.105022 & 2.202524 & -.433227 \\ 65 & 6 & 0 & -5.848966 & -.398507 & -.741978 \\ 66 & 6 & 0 & -5.043950 & -2.637297 & -1.293662 \\ 67 & 1 & 0 & -2.924356 & -2.832628 & -1.705540 \\ 68 & 6 & 0 & 5.316319 & -.770587 & 3.237235 \\ 69 & 1 & 0 & 3.349047 & -.000472 & 3.706995 \\ 70 & 1 & 0 & 2.105847 & 2.842055 & -3.563673 \\ 71 & 1 & 0 & .957059 & 4.136022 & -3.958524 \\ 73 & 1 & 0 & 2.300762 & 4.455867 & -2.842273 \\ 75 & 1 & 0 & -1.366787 & 5.005535 & -2.189982 \\ & 1 & 0 & -1.338394 & 4.752495 & -.429093 \\ 73 & 0 & -.063682 & 5.708354 & -1.209709\end{array}$




$\begin{array}{cccccc}76 & 1 & 0 & -5.297115 & -2.375748 & 2.578211 \\ 77 & 6 & 0 & -5.277979 & -.212955 & 2.841784 \\ 78 & 1 & 0 & -4.946377 & 1.931049 & 3.015850 \\ 79 & 1 & 0 & 4.150963 & -2.727546 & -3.304984 \\ 80 & 6 & 0 & 2.529083 & -4.155656 & -3.047967 \\ 81 & 1 & 0 & .811054 & -5.398028 & -2.547114 \\ 82 & 6 & 0 & -6.103723 & -1.755035 & -.943186 \\ 83 & 1 & 0 & -6.665321 & .285049 & -.471641 \\ 84 & 1 & 0 & -5.253131 & -3.704127 & -1.449619 \\ 85 & 6 & 0 & 6.325526 & -.913878 & 2.243331 \\ 86 & 1 & 0 & 6.913351 & -.483476 & .203286 \\ 87 & 1 & 0 & 5.480957 & -1.180313 & 4.242719 \\ 88 & 1 & 0 & -6.329049 & -.160119 & 3.154237 \\ 89 & 1 & 0 & 2.872115 & -4.814856 & -3.856127 \\ 90 & 1 & 0 & -7.123506 & -2.146977 & -.831668 \\ 91 & 1 & 0 & 7.259779 & -1.436391 & 2.488045 \\ ---------------------------------------------------------------\end{array}$

\section{envelope (rac-3)}

absolute energy: -1899.90177880 hartrees imaginary frequencies: 0

\begin{tabular}{|c|c|c|c|c|c|}
\hline \multirow{2}{*}{$\begin{array}{l}\text { Center } \\
\text { Number }\end{array}$} & \multirow{2}{*}{$\begin{array}{l}\text { Atomic } \\
\text { Number }\end{array}$} & \multirow{2}{*}{\multicolumn{2}{|c|}{$\begin{array}{r}\text { Atomic } \\
\text { Type }\end{array}$}} & \multicolumn{2}{|c|}{ Coordinates (Angstroms } \\
\hline & & & & $\mathrm{X}$ & $\mathrm{Z}$ \\
\hline 1 & 40 & 0 & -.000192 & -.000131 & .153008 \\
\hline 2 & 7 & 0 & -1.120859 & -.891255 & -1.345878 \\
\hline 3 & 7 & 0 & 1.121033 & .892553 & -1.344350 \\
\hline 4 & 6 & 0 & .740291 & -1.190696 & 2.421153 \\
\hline 5 & 6 & 0 & .018344 & -2.223327 & 1.718447 \\
\hline 6 & 6 & 0 & .759171 & -2.578111 & .533154 \\
\hline 7 & 6 & 0 & 1.972607 & -1.813023 & .499044 \\
\hline 8 & 6 & 0 & 1.959341 & -.933124 & 1.664314 \\
\hline 9 & 6 & 0 & -.739662 & 1.188362 & 2.422366 \\
\hline 10 & 6 & 0 & -.017945 & 2.221567 & 1.720331 \\
\hline 11 & 6 & 0 & -.759050 & 2.577300 & .535539 \\
\hline 12 & 6 & 0 & -1.972515 & 1.812222 & .501099 \\
\hline 13 & 6 & 0 & -1.958989 & .931460 & 1.665711 \\
\hline 14 & 6 & 0 & 1.305440 & .080548 & -2.627471 \\
\hline 15 & 6 & 0 & -1.303470 & -.078739 & -2.628946 \\
\hline 16 & 6 & 0 & -1.755771 & -2.215085 & -1.489135 \\
\hline 17 & 6 & 0 & 1.754760 & 2.217116 & -1.487163 \\
\hline 18 & 1 & 0 & .523327 & -3.432065 & -.109275 \\
\hline 19 & 6 & 0 & -1.163727 & -3.048481 & 2.242389 \\
\hline 20 & 6 & 0 & 3.158466 & -.163428 & 1.985742 \\
\hline 21 & 6 & 0 & 1.165342 & 3.045108 & 2.244220 \\
\hline
\end{tabular}




\begin{tabular}{llllll}
22 & 6 & 0 & -3.158064 & .161523 & 1.986777 \\
23 & 6 & 0 & -3.176101 & 1.934269 & -.336060 \\
24 & 6 & 0 & 3.176044 & -1.934445 & -.338412 \\
25 & 1 & 0 & -.523462 & 3.431835 & -.106199 \\
26 & 6 & 0 & .001473 & .001015 & -3.494779 \\
27 & 1 & 0 & 2.125908 & .528235 & -3.222589 \\
28 & 1 & 0 & 1.615630 & -.940959 & -2.349960 \\
29 & 1 & 0 & -1.613853 & .942695 & -2.351341 \\
30 & 1 & 0 & -2.123296 & -.526056 & -3.225213 \\
31 & 14 & 0 & .000867 & -.001522 & 3.769164 \\
32 & 6 & 0 & -1.304946 & -3.138018 & -2.474773 \\
33 & 6 & 0 & -2.850390 & -2.578528 & -.664204 \\
34 & 6 & 0 & 2.851345 & 2.579903 & -.664592 \\
35 & 6 & 0 & 1.300827 & 3.141154 & -2.470290 \\
36 & 1 & 0 & -.074350 & .887990 & -4.146295 \\
37 & 1 & 0 & .078035 & -.885849 & -4.146365 \\
38 & 6 & 0 & 1.332556 & .822752 & 4.983257 \\
39 & 6 & 0 & -1.329000 & -.825902 & 4.985152 \\
40 & 6 & 0 & 4.328355 & -1.150725 & .026124 \\
41 & 6 & 0 & 4.279550 & -.280054 & 1.203117 \\
42 & 1 & 0 & 3.172439 & .456653 & 2.890463 \\
43 & 6 & 0 & 3.261403 & -2.818923 & -1.455851 \\
44 & 6 & 0 & -4.279345 & .278855 & 1.204540 \\
45 & 6 & 0 & -4.328387 & 1.150388 & .028204 \\
46 & 1 & 0 & -3.171852 & -.459442 & 2.890893 \\
47 & 6 & 0 & -3.261618 & 2.819437 & -1.452935 \\
48 & 1 & 0 & -.843741 & -3.628308 & 3.126730 \\
49 & 1 & 0 & -2.019913 & -2.427287 & 2.534019 \\
50 & 1 & 0 & -1.500976 & -3.751823 & 1.467671 \\
51 & 1 & 0 & 1.492986 & 3.758907 & 1.474925 \\
52 & 1 & 0 & .850795 & 3.613129 & 3.138042 \\
53 & 1 & 0 & 2.026652 & 2.424054 & 2.520870 \\
54 & 1 & 0 & -.456837 & -2.870310 & -3.118383 \\
55 & 6 & 0 & -1.927558 & -4.389714 & -2.615343 \\
56 & 1 & 0 & -3.221846 & -1.855000 & .074826 \\
57 & 6 & 0 & -3.465639 & -3.838125 & -.809359 \\
58 & 1 & 0 & 3.225056 & 1.855613 & .072553 \\
59 & 6 & 0 & 3.465514 & 3.840046 & -.809552 \\
60 & 1 & 0 & .451216 & 2.873783 & -3.112074 \\
61 & 6 & 0 & 1.922403 & 4.393389 & -2.610737 \\
62 & 6 & 0 & 5.515871 & -1.263832 & -.762060 \\
63 & 1 & 0 & 5.192986 & .271674 & 1.463830 \\
64 & 1 & 0 & -5.192712 & -.273097 & 1.465016 \\
67 & 6 & 0 & -5.516065 & 1.264074 & -.759651 \\
& 6 & 0 & -4.437626 & 2.912518 & -2.200947 \\
\hline 6 & 0 & -2.387413 & 3.427951 & -1.720755
\end{tabular}




$\begin{array}{lllccl}68 & 6 & 0 & 4.437266 & -2.911469 & -2.204156 \\ 69 & 1 & 0 & 2.387172 & -3.427309 & -1.723881 \\ 70 & 1 & 0 & 2.057799 & 1.450917 & 4.458938 \\ 71 & 1 & 0 & .812435 & 1.442852 & 5.722914 \\ 72 & 1 & 0 & 1.876009 & .035518 & 5.519170 \\ 73 & 1 & 0 & -2.059436 & -1.448387 & 4.461263 \\ 74 & 1 & 0 & -.808395 & -1.451741 & 5.719628 \\ 75 & 1 & 0 & -1.866545 & -.038731 & 5.527095 \\ 76 & 1 & 0 & -1.564791 & -5.094067 & -3.376281 \\ 77 & 6 & 0 & -3.008111 & -4.746828 & -1.782145 \\ 78 & 1 & 0 & -4.314036 & -4.104195 & -.164338 \\ 79 & 1 & 0 & 4.315398 & 4.105708 & -.166322 \\ 80 & 6 & 0 & 3.004931 & 4.749877 & -1.779837 \\ 81 & 1 & 0 & 1.557292 & 5.098623 & -3.369736 \\ 82 & 6 & 0 & -5.570112 & 2.125599 & -1.856776 \\ 83 & 1 & 0 & -6.392853 & .661848 & -.484794 \\ 84 & 1 & 0 & -4.489528 & 3.594993 & -3.059922 \\ 85 & 6 & 0 & 5.569757 & -2.124670 & -1.859734 \\ 86 & 1 & 0 & 6.392680 & -.661725 & -.487010 \\ 87 & 1 & 0 & 4.489038 & -3.593420 & -3.063555 \\ 88 & 1 & 0 & -3.490795 & -5.726326 & -1.894551 \\ 89 & 1 & 0 & 3.486765 & 5.729813 & -1.892090 \\ 90 & 1 & 0 & -6.488863 & 2.202557 & -2.453181 \\ 91 & 1 & 0 & 6.488395 & -2.201189 & -2.456370 \\ ------------------------------------------------------------\end{array}$

\section{twist (rac-3) \\ absolute energy: $\mathbf{- 1 8 9 9 . 8 9 3 8 0 5 6 2}$ hartrees imaginary frequencies: 0}

\begin{tabular}{|c|c|c|c|c|c|}
\hline \multirow{2}{*}{$\begin{array}{l}\text { Center } \\
\text { Number }\end{array}$} & \multirow{2}{*}{$\begin{array}{l}\text { Atomic } \\
\text { Number }\end{array}$} & \multirow{2}{*}{\multicolumn{2}{|c|}{$\begin{array}{r}\text { Atomic } \\
\text { Type }\end{array}$}} & \multicolumn{2}{|c|}{ Coordinates (Angstrom } \\
\hline & & & & $\mathrm{X}$ & $\mathrm{Z}$ \\
\hline 1 & 40 & 0 & .509847 & -.384224 & -.153133 \\
\hline 2 & 7 & 0 & 1.665385 & -.331427 & -1.868871 \\
\hline 3 & 7 & 0 & -.672558 & 1.111725 & -.986707 \\
\hline 4 & 6 & 0 & -.329532 & -2.519383 & 1.196314 \\
\hline 5 & 6 & 0 & .212115 & -2.963576 & -.071979 \\
\hline 6 & 6 & 0 & -.695908 & -2.574874 & -1.123498 \\
\hline 7 & 6 & 0 & -1.847989 & -1.959779 & -.523307 \\
\hline 8 & 6 & 0 & -1.610344 & -1.890926 & .914271 \\
\hline 9 & 6 & 0 & 1.561107 & -.746509 & 2.273016 \\
\hline 10 & 6 & 0 & 2.711479 & -.652073 & 1.414602 \\
\hline 11 & 6 & 0 & 2.761361 & .679583 & .852579 \\
\hline 12 & 6 & 0 & 1.691771 & 1.456159 & 1.409822 \\
\hline 13 & 6 & 0 & .918002 & .567177 & 2.270110 \\
\hline
\end{tabular}




$\begin{array}{llllll}14 & 6 & 0 & .860272 & -.140327 & -3.152799 \\ 15 & 6 & 0 & .038899 & 2.038520 & -1.977871 \\ 16 & 6 & 0 & 3.108731 & -.344182 & -2.184525 \\ 17 & 6 & 0 & -2.079434 & 1.518327 & -.829003 \\ 18 & 1 & 0 & -.635961 & -2.951800 & -2.148799 \\ 19 & 6 & 0 & 1.364284 & -3.950842 & -.300398 \\ 20 & 6 & 0 & -2.703725 & -1.496626 & 1.797000 \\ 21 & 6 & 0 & 3.885792 & -1.631175 & 1.315964 \\ 22 & 6 & 0 & -.131407 & 1.135445 & 3.112280 \\ 23 & 6 & 0 & 1.421778 & 2.901351 & 1.377198 \\ 24 & 6 & 0 & -3.177026 & -1.675298 & -1.080221 \\ 25 & 1 & 0 & 3.610941 & 1.076522 & .288955 \\ 26 & 14 & 0 & .686177 & -2.388112 & 2.848670 \\ 27 & 1 & 0 & -.030016 & -.790113 & -3.104563 \\ 28 & 1 & 0 & 1.469824 & -.460995 & -4.021672 \\ 29 & 6 & 0 & .389340 & 1.344198 & -3.338227 \\ 30 & 1 & 0 & -.603272 & 2.921032 & -2.164701 \\ 31 & 1 & 0 & .969758 & 2.401623 & -1.509212 \\ 32 & 6 & 0 & 3.828361 & -1.566831 & -2.183975 \\ 33 & 6 & 0 & 3.792642 & .851968 & -2.535441 \\ 34 & 6 & 0 & -2.621262 & 1.747172 & .461450 \\ 35 & 6 & 0 & -2.908951 & 1.745151 & -1.965915 \\ 36 & 6 & 0 & 1.887149 & -3.923628 & 3.210645 \\ 37 & 6 & 0 & -.394261 & -2.218549 & 4.500761 \\ 38 & 6 & 0 & -4.232099 & -1.347141 & -.156523 \\ 39 & 6 & 0 & -3.949855 & -1.256252 & 1.277184 \\ 40 & 1 & 0 & -2.534771 & -1.458737 & 2.879727 \\ 41 & 6 & 0 & -3.479593 & -1.793912 & -2.470965 \\ 42 & 6 & 0 & -.365639 & 2.487833 & 3.097520 \\ 43 & 6 & 0 & .382779 & 3.406281 & 2.237243 \\ 44 & 1 & 0 & -.687944 & .481973 & 3.794245 \\ 45 & 6 & 0 & 2.171551 & 3.816655 & .578363 \\ 46 & 1 & 0 & 1.101240 & -4.929523 & .140392 \\ 47 & 1 & 0 & 1.523199 & -4.091447 & -1.379685 \\ 48 & 1 & 0 & 2.309882 & -3.619218 & .147016 \\ 49 & 1 & 0 & 4.681025 & -1.308982 & 2.013220 \\ 50 & 1 & 0 & 3.589891 & -2.650251 & 1.592125 \\ 51 & 1 & 0 & 4.307512 & -1.644122 & .300815 \\ 52 & 1 & 0 & 1.181994 & 1.933009 & -3.830457 \\ 53 & 1 & 0 & -.491806 & 1.343568 & -4.002207 \\ 54 & 1 & 0 & 3.298334 & -2.496449 & -1.937404 \\ 55 & 6 & 0 & 5.198921 & -1.590023 & -2.511484 \\ 56 & 1 & 0 & 3.246703 & 1.804706 & -2.536647 \\ 57 & 6 & 0 & 5.159892 & .820249 & -2.863536 \\ 58 & 1 & 0 & -1.977178 & 1.617324 & 1.340900 \\ 59 & 6 & 0 & -3.951443 & 2.185372 & .611881\end{array}$




$\begin{array}{rrrrrl}60 & 1 & 0 & -2.513140 & 1.564144 & -2.973049 \\ 61 & 6 & 0 & -4.232268 & 2.186041 & -1.805227 \\ 62 & 6 & 0 & -5.551932 & -1.137944 & -.666898 \\ 63 & 1 & 0 & -4.785093 & -1.000620 & 1.942821 \\ 64 & 1 & 0 & -1.130943 & 2.920401 & 3.756169 \\ 65 & 6 & 0 & .116959 & 4.811326 & 2.244316 \\ 66 & 6 & 0 & 1.891067 & 5.184394 & .609138 \\ 67 & 1 & 0 & 2.973746 & 3.431296 & -.065123 \\ 68 & 6 & 0 & -4.778379 & -1.587470 & -2.938612 \\ 69 & 1 & 0 & -2.674684 & -2.049381 & -3.172948 \\ 70 & 1 & 0 & 1.299302 & -4.737876 & 3.650981 \\ 71 & 1 & 0 & 2.372964 & -4.303650 & 2.307905 \\ 72 & 1 & 0 & 2.661194 & -3.633827 & 3.930826 \\ 73 & 1 & 0 & -1.130355 & -1.411211 & 4.452322 \\ 74 & 1 & 0 & -.926148 & -3.159475 & 4.685327 \\ 75 & 1 & 0 & .270249 & -2.028431 & 5.352144 \\ 76 & 1 & 0 & 5.742392 & -2.544665 & -2.506424 \\ 77 & 6 & 0 & 5.868304 & -.398791 & -2.850792 \\ 78 & 1 & 0 & 5.676923 & 1.753025 & -3.127084 \\ 79 & 1 & 0 & -4.348752 & 2.367778 & 1.619445 \\ 80 & 6 & 0 & -4.761085 & 2.409205 & -.516807 \\ 81 & 1 & 0 & -4.858228 & 2.353004 & -2.692237 \\ 82 & 6 & 0 & .853776 & 5.684716 & 1.442838 \\ 83 & 1 & 0 & -.680003 & 5.195816 & 2.895421 \\ 84 & 1 & 0 & 2.471923 & 5.878602 & -.012943 \\ 85 & 6 & 0 & -5.821627 & -1.256322 & -2.030782 \\ 86 & 1 & 0 & -6.356541 & -.885039 & .036922 \\ 87 & 1 & 0 & -4.998527 & -1.681191 & -4.010430 \\ 88 & 1 & 0 & 6.935988 & -.418506 & -3.105662 \\ 89 & 1 & 0 & -5.794770 & 2.758363 & -.397426 \\ 90 & 1 & 0 & .638067 & 6.761316 & 1.457165 \\ 91 & 1 & 0 & -6.840549 & -1.095672 & -2.407431 \\ -----------------------------------------------------------\end{array}$

\section{meso-(MBSBI)Zr $\left\{\mathrm{PhN}\left(\mathrm{CH}_{2}\right)_{3} \mathrm{NPh}\right\}$ (3) boat (meso-3) \\ absolute energy: -1899.89081349 hartrees imaginary frequencies: 0}

\begin{tabular}{lrrrrr} 
Center & Atomic & \multicolumn{2}{c}{ Atomic } & \multicolumn{2}{c}{ Coordinates (Angstrom } \\
Number & Number & Type & X & Y & Z \\
- \hdashline 1 & 40 & 0 & .484219 & -.492047 & -.163101 \\
2 & 7 & 0 & 1.816784 & -.801172 & -1.708428 \\
3 & 7 & 0 & -.720400 & .697278 & -1.377825
\end{tabular}




$\begin{array}{llllll}4 & 6 & 0 & -.304017 & -2.430718 & 1.570974 \\ 5 & 6 & 0 & .363715 & -3.058522 & .461945 \\ 6 & 6 & 0 & -.492282 & -2.969729 & -.703161 \\ 7 & 6 & 0 & -1.749455 & -2.394624 & -.298163 \\ 8 & 6 & 0 & -1.620209 & -2.006930 & 1.095513 \\ 9 & 6 & 0 & 1.264825 & -.225274 & 2.307926 \\ 10 & 6 & 0 & 2.497753 & -.132733 & 1.563828 \\ 11 & 6 & 0 & 2.465190 & 1.065811 & .764695 \\ 12 & 6 & 0 & 1.271518 & 1.806222 & 1.091543 \\ 13 & 6 & 0 & .507371 & .998695 & 2.029489 \\ 14 & 6 & 0 & 1.309753 & -1.246303 & -3.082989 \\ 15 & 6 & 0 & -.494220 & .636456 & -2.889055 \\ 16 & 6 & 0 & 3.287827 & -.639587 & -1.833374 \\ 17 & 6 & 0 & -1.678651 & 1.767236 & -1.063097 \\ 18 & 1 & 0 & -.329445 & -3.556998 & -1.611994 \\ 19 & 6 & 0 & 1.620274 & -3.937966 & .495801 \\ 20 & 6 & 0 & -2.791668 & -1.536217 & 1.818556 \\ 21 & 6 & 0 & 3.766427 & -.966520 & 1.766436 \\ 22 & 6 & 0 & -.659479 & 1.583355 & 2.682108 \\ 23 & 6 & 0 & .907664 & 3.202975 & .823035 \\ 24 & 6 & 0 & -3.046727 & -2.321903 & -.977183 \\ 25 & 1 & 0 & 3.325859 & 1.463661 & .219416 \\ 26 & 14 & 0 & .561539 & -1.811195 & 3.195047 \\ 27 & 6 & 0 & -.150043 & -.794134 & -3.395626 \\ 28 & 6 & 0 & 4.156093 & -1.721593 & -1.541571 \\ 29 & 6 & 0 & 3.836344 & .572663 & -2.328374 \\ 30 & 6 & 0 & -2.713536 & 1.555963 & -.114616 \\ 31 & 6 & 0 & -1.620196 & 3.026419 & -1.731066 \\ 32 & 1 & 0 & 1.390033 & -2.348708 & -3.173822 \\ 33 & 1 & 0 & 1.987157 & -.792459 & -3.834619 \\ 34 & 1 & 0 & .306058 & 1.341339 & -3.192028 \\ 35 & 1 & 0 & -1.437186 & .952507 & -3.378461 \\ 36 & 6 & 0 & 1.922875 & -3.045351 & 3.940080 \\ 37 & 6 & 0 & -.637848 & -1.434879 & 4.727094 \\ 38 & 6 & 0 & -4.178348 & -1.853748 & -.215230 \\ 39 & 6 & 0 & -4.009031 & -1.471074 & 1.184802 \\ 40 & 1 & 0 & -2.704041 & -1.277808 & 2.880499 \\ 41 & 6 & 0 & -3.252264 & -2.751972 & -2.325366 \\ 42 & 6 & 0 & -.979581 & 2.899093 & 2.455112 \\ 43 & 6 & 0 & -.216345 & 3.746916 & 1.539996 \\ 44 & 1 & 0 & -1.232061 & .986156 & 3.400818 \\ 45 & 6 & 0 & 1.656468 & 4.049200 & -.050452 \\ 47 & 1 & 0 & 1.883913 & -4.247368 & -.526306 \\ & 1 & 0 & 2.489718 & -3.431324 & .934861 \\ 4 & 1 & 0 & 1.419868 & -4.847839 & 1.090471 \\ 4 & 0 & 4.404487 & -.465794 & 2.518004\end{array}$




$\begin{array}{cccccc}50 & 1 & 0 & 3.538232 & -1.973319 & 2.134969 \\ 51 & 1 & 0 & 4.338387 & -1.051449 & .832299 \\ 52 & 1 & 0 & 3.730213 & -2.667347 & -1.180144 \\ 53 & 6 & 0 & 5.547624 & -1.583041 & -1.720257 \\ 54 & 1 & 0 & 3.160749 & 1.405669 & -2.568781 \\ 55 & 6 & 0 & 5.226300 & .704075 & -2.501122 \\ 56 & 1 & 0 & -2.802655 & .579229 & .377622 \\ 57 & 6 & 0 & -3.654131 & 2.566909 & .157223 \\ 58 & 1 & 0 & -.821519 & 3.222262 & -2.456373 \\ 59 & 6 & 0 & -2.568504 & 4.025282 & -1.457485 \\ 60 & 1 & 0 & -.274822 & -.813892 & -4.492974 \\ 61 & 1 & 0 & -.876939 & -1.499678 & -2.966651 \\ 62 & 6 & 0 & -5.461683 & -1.799301 & -.846711 \\ 63 & 1 & 0 & -4.900423 & -1.140005 & 1.733953 \\ 64 & 1 & 0 & -1.829467 & 3.355584 & 2.980405 \\ 65 & 6 & 0 & -.552793 & 5.124755 & 1.348447 \\ 66 & 6 & 0 & 1.304091 & 5.389241 & -.215866 \\ 67 & 1 & 0 & 2.512753 & 3.630206 & -.595587 \\ 68 & 6 & 0 & -4.516145 & -2.689901 & -2.912450 \\ 69 & 1 & 0 & -2.400793 & -3.139332 & -2.899611 \\ 70 & 1 & 0 & 1.418135 & -3.789698 & 4.567480 \\ 71 & 1 & 0 & 2.481611 & -3.575984 & 3.164304 \\ 72 & 1 & 0 & 2.631128 & -2.493340 & 4.568732 \\ 73 & 1 & 0 & -1.441426 & -.734502 & 4.483176 \\ 74 & 1 & 0 & -1.089309 & -2.372116 & 5.073903 \\ 75 & 1 & 0 & -.051167 & -1.012030 & 5.551469 \\ 76 & 1 & 0 & 6.211628 & -2.426893 & -1.488418 \\ 77 & 6 & 0 & 6.085491 & -.371960 & -2.196522 \\ 78 & 1 & 0 & 5.640973 & 1.649304 & -2.877101 \\ 79 & 1 & 0 & -4.454253 & 2.375609 & .884942 \\ 80 & 6 & 0 & -3.591342 & 3.803095 & -.513313 \\ 81 & 1 & 0 & -2.505711 & 4.987581 & -1.983357 \\ 82 & 6 & 0 & .192404 & 5.930884 & .488081 \\ 83 & 1 & 0 & -1.411840 & 5.539614 & 1.893158 \\ 84 & 1 & 0 & 1.885235 & 6.031309 & -.891359 \\ 85 & 6 & 0 & -5.628438 & -2.202987 & -2.170783 \\ 86 & 1 & 0 & -6.320384 & -1.435328 & -.266199 \\ 87 & 1 & 0 & -4.657041 & -3.020286 & -3.950248 \\ 88 & 1 & 0 & 7.169747 & -.266456 & -2.333384 \\ 89 & 1 & 0 & -4.333699 & 4.584698 & -.307180 \\ 90 & 1 & 0 & -.076233 & 6.986761 & .350648 \\ 91 & 1 & 0 & -6.618868 & -2.155322 & -2.642477 \\ ---------------------------------------------------------------- \\ 53\end{array}$

\section{chair (meso-3)}

absolute energy: $-\mathbf{1 8 9 9 . 8 9 2 5 4 8 2 0}$ hartrees 


\section{imaginary frequencies: 0}

\begin{tabular}{|c|c|c|c|c|c|}
\hline \multirow{3}{*}{$\begin{array}{l}\text { Center } \\
\text { Number } \\
-0 .-\end{array}$} & \multirow{2}{*}{$\begin{array}{l}\text { Atomic } \\
\text { Number }\end{array}$} & \multirow{2}{*}{\multicolumn{2}{|c|}{$\begin{array}{l}\text { Atomic } \\
\text { Type }\end{array}$}} & \multicolumn{2}{|c|}{ Coordinates (Angstroms } \\
\hline & & & & $\mathrm{X}$ & $\mathrm{Z}$ \\
\hline & 40 & 0 & .530803 & -.501034 & -.140415 \\
\hline 2 & 7 & 0 & 1.873919 & -.715839 & -1.694913 \\
\hline 3 & 7 & 0 & -.882358 & .464407 & -1.297754 \\
\hline 4 & 6 & 0 & -.064238 & -2.468122 & 1.576932 \\
\hline 5 & 6 & 0 & .634807 & -3.074463 & .469249 \\
\hline 6 & 6 & 0 & -.243911 & -3.082639 & -.678529 \\
\hline 7 & 6 & 0 & -1.534314 & -2.590890 & -.266692 \\
\hline 8 & 6 & 0 & -1.416934 & -2.162697 & 1.114783 \\
\hline 9 & 6 & 0 & 1.254980 & -.093436 & 2.287938 \\
\hline 10 & 6 & 0 & 2.467791 & .137052 & 1.528254 \\
\hline 11 & 6 & 0 & 2.297874 & 1.323830 & .739305 \\
\hline 12 & 6 & 0 & 1.030041 & 1.927949 & 1.079457 \\
\hline 13 & 6 & 0 & .373800 & 1.047302 & 2.032670 \\
\hline 14 & 6 & 0 & 1.298920 & -1.092353 & -3.062239 \\
\hline 15 & 6 & 0 & -1.0521 & -.027585 & -2.741282 \\
\hline 16 & 6 & 0 & 3.319662 & -.438599 & -1.845739 \\
\hline 17 & 6 & 0 & -1.787260 & 1.605840 & -1.061164 \\
\hline 18 & 1 & 0 & -.052446 & -3.677083 & -1.577288 \\
\hline 19 & 6 & 0 & 1.937559 & -3.883336 & .517386 \\
\hline 20 & 6 & 0 & -2.603541 & -1.721610 & 1.828021 \\
\hline 21 & 6 & 0 & 3.814834 & -.574532 & 1.702392 \\
\hline 22 & 6 & 0 & -.828014 & 1.510941 & 2.717397 \\
\hline 23 & 6 & 0 & .533203 & 3.288713 & .841193 \\
\hline 24 & 6 & 0 & -2.836336 & -2.606342 & -.938010 \\
\hline 25 & 1 & 0 & 3.102100 & 1.812489 & .181322 \\
\hline 26 & 14 & 0 & .758824 & -1.749346 & 3.182526 \\
\hline 27 & 1 & 0 & .814479 & -2.084760 & -2.994620 \\
\hline 28 & 1 & 0 & 2.137536 & -1.167167 & -3.782700 \\
\hline 29 & 6 & 0 & .259040 & -.054527 & -3.581945 \\
\hline 30 & 1 & 0 & -1.494879 & -1.042049 & -2.727656 \\
\hline 31 & 1 & 0 & -1.778345 & .644596 & -3.237168 \\
\hline 32 & 6 & 0 & 4.275783 & -1.428918 & -1.505899 \\
\hline 33 & 6 & 0 & 3.770714 & .792294 & -2.394830 \\
\hline 34 & 6 & 0 & -2.852102 & 1.503406 & -.130453 \\
\hline 35 & 6 & 0 & -1.653364 & 2.801234 & -1.823604 \\
\hline 36 & 6 & 0 & 2.287314 & -2.799321 & 3.877862 \\
\hline 37 & 6 & 0 & -.444892 & -1.534259 & 4.742147 \\
\hline 38 & 6 & 0 & -3.984885 & -2.165974 & -.185589 \\
\hline 39 & 6 & 0 & -3.826115 & -1.728028 & 1.198456 \\
\hline 40 & 1 & 0 & -2.523706 & -1.424016 & 2.880383 \\
\hline 41 & 6 & 0 & -3.023435 & -3.083109 & -2.273908 \\
\hline
\end{tabular}




$\begin{array}{llllll}42 & 6 & 0 & -1.275312 & 2.792921 & 2.515181 \\ 43 & 6 & 0 & -.614755 & 3.719193 & 1.596174 \\ 44 & 1 & 0 & -1.323337 & .855436 & 3.442890 \\ 45 & 6 & 0 & 1.184547 & 4.213702 & -.030740 \\ 46 & 1 & 0 & 2.213567 & -4.204987 & -.497743 \\ 47 & 1 & 0 & 2.781663 & -3.323156 & .940667 \\ 48 & 1 & 0 & 1.787680 & -4.785662 & 1.137869 \\ 49 & 1 & 0 & 4.161223 & -.445067 & 2.743010 \\ 50 & 1 & 0 & 3.762159 & -1.650577 & 1.493951 \\ 51 & 1 & 0 & 4.560397 & -.132118 & 1.026455 \\ 52 & 1 & 0 & .715929 & .950025 & -3.594189 \\ 53 & 1 & 0 & -.009957 & -.326166 & -4.619149 \\ 54 & 1 & 0 & 3.926643 & -2.388633 & -1.102303 \\ 55 & 6 & 0 & 5.651235 & -1.185853 & -1.691550 \\ 56 & 1 & 0 & 3.034124 & 1.560318 & -2.667462 \\ 57 & 6 & 0 & 5.145389 & 1.027022 & -2.578705 \\ 58 & 1 & 0 & -2.982421 & .569817 & .433944 \\ 59 & 6 & 0 & -3.757884 & 2.570824 & .033604 \\ 60 & 1 & 0 & -.827877 & 2.891209 & -2.542411 \\ 61 & 6 & 0 & -2.565102 & 3.856780 & -1.656725 \\ 62 & 6 & 0 & -5.271457 & -2.191140 & -.814357 \\ 63 & 1 & 0 & -4.728773 & -1.420208 & 1.742649 \\ 64 & 1 & 0 & -2.150183 & 3.162850 & 3.066710 \\ 65 & 6 & 0 & -1.073013 & 5.066311 & 1.441808 \\ 66 & 6 & 0 & .715681 & 5.521758 & -.157530 \\ 67 & 1 & 0 & 2.059340 & 3.880726 & -.604965 \\ 68 & 6 & 0 & -4.289935 & -3.098817 & -2.857104 \\ 69 & 1 & 0 & -2.153168 & -3.441098 & -2.839967 \\ 70 & 1 & 0 & 3.030786 & -3.025566 & 3.109823 \\ 71 & 1 & 0 & 2.778783 & -2.244733 & 4.685781 \\ 72 & 1 & 0 & 1.916926 & -3.745649 & 4.289824 \\ 73 & 1 & 0 & -1.318487 & -.913778 & 4.525030 \\ 74 & 1 & 0 & -.794603 & -2.519947 & 5.071704 \\ 75 & 1 & 0 & .108822 & -1.072645 & 5.568384 \\ 76 & 1 & 0 & 6.381320 & -1.960984 & -1.421917 \\ 77 & 6 & 0 & 6.089578 & .040976 & -2.225990 \\ 78 & 1 & 0 & 5.481847 & 1.984833 & -2.998388 \\ 79 & 1 & 0 & -4.584610 & 2.472807 & .750025 \\ 80 & 6 & 0 & -3.621342 & 3.745948 & -.728346 \\ 81 & 1 & 0 & -2.449852 & 4.773200 & -2.251061 \\ 82 & 6 & 0 & -.420500 & 5.951195 & .583665 \\ 83 & 1 & 0 & -1.950260 & 5.394930 & 2.015524 \\ 84 & 1 & 0 & 1.223583 & 6.225157 & -.830894 \\ 85 & 6 & 0 & -5.422130 & -2.642741 & -2.124337 \\ & 1 & 0 & -6.144776 & -1.848738 & -.242575 \\ & 1 & 0 & -4.418374 & -3.464332 & -3.884645\end{array}$




$\begin{array}{llllll}88 & 1 & 0 & 7.161758 & .228468 & -2.369609 \\ 89 & 1 & 0 & -4.334126 & 4.571533 & -.604329 \\ 90 & 1 & 0 & -.781162 & 6.982928 & .476910 \\ 91 & 1 & 0 & -6.414796 & -2.655395 & -2.593566\end{array}$

\section{envelope (meso-3)}

absolute energy: $-\mathbf{1 8 9 9 . 8 9 0 5 8 0 2 2}$ hartrees imaginary frequencies: 0

\begin{tabular}{|c|c|c|c|c|c|}
\hline \multirow{2}{*}{$\begin{array}{l}\text { Center } \\
\text { Number }\end{array}$} & \multirow{2}{*}{\multicolumn{2}{|c|}{$\begin{array}{l}\text { Atomic } \\
\text { Number }\end{array}$}} & \multirow{2}{*}{$\begin{array}{r}\text { Atomic } \\
\text { Type }\end{array}$} & \multicolumn{2}{|c|}{ Coordinates (Angstroms } \\
\hline & & & & $X$ & $\mathrm{Z}$ \\
\hline 1 & 40 & 0 & -.483366 & -.493635 & -.162775 \\
\hline 2 & 7 & 0 & -1.812727 & -.813488 & -1.708198 \\
\hline 3 & 7 & 0 & .711988 & .704469 & -1.377951 \\
\hline 4 & 6 & 0 & -1.266246 & -.240548 & 2.310062 \\
\hline 5 & 6 & 0 & -2.499878 & -.156436 & 1.566666 \\
\hline 6 & 6 & 0 & -2.476388 & 1.043326 & .768582 \\
\hline 7 & 6 & 0 & -1.288174 & 1.792293 & 1.095913 \\
\hline 8 & 6 & 0 & -.517576 & 989068 & 2.032242 \\
\hline 9 & 6 & 0 & .326194 & -2.427362 & 1.567036 \\
\hline 10 & 6 & 0 & -.337658 & -3.057319 & .456568 \\
\hline 11 & 6 & 0 & .518517 & -2.962323 & -.707776 \\
\hline 12 & 6 & 0 & 1.772192 & -2.380832 & $2 \quad-.301256$ \\
\hline 13 & 6 & 0 & 1.640539 & -1.996452 & 1.093075 \\
\hline 14 & 6 & 0 & -1.301729 & -1.257290 & $\begin{array}{ll}0 & -3.081929\end{array}$ \\
\hline 15 & 6 & 0 & .479639 & .647887 & -2.888422 \\
\hline 16 & 6 & 0 & 1.664828 & 1.778920 & ) -1.062292 \\
\hline 17 & 6 & 0 & -3.284247 & -.657362 & -1.834425 \\
\hline 18 & 1 & 0 & -3.340371 & 1.436029 & .224857 \\
\hline 19 & 6 & 0 & -3.762006 & -1.000664 & 1.767127 \\
\hline 20 & 6 & 0 & .645986 & 1.580968 & 2.684355 \\
\hline 21 & 6 & 0 & -1.589582 & -3.943442 & .489111 \\
\hline 22 & 6 & 0 & 2.809187 & -1.520038 & $8 \quad 1.816772$ \\
\hline 23 & 6 & 0 & 3.068477 & -2.297966 & -.980634 \\
\hline 24 & 6 & 0 & -.934385 & 3.191960 & .828825 \\
\hline 25 & 1 & 0 & .359546 & -3.549489 & -1.617364 \\
\hline 26 & 14 & 0 & -.545830 & -1.821184 & 3.192544 \\
\hline 27 & 1 & 0 & -1.365857 & -2.361045 & $5 \quad-3.167562$ \\
\hline 28 & 1 & 0 & -1.987037 & -.816903 & -3.834339 \\
\hline 29 & 6 & 0 & .151149 & -.785050 & -3.398928 \\
\hline 30 & 1 & 0 & 1.416490 & .977378 & -3.380674 \\
\hline 31 & 1 & 0 & -.330462 & 1.344161 & -3.185059 \\
\hline 32 & 6 & 0 & 2.702953 & 1.570497 & $7 \quad-.116705$ \\
\hline 33 & 6 & 0 & 1.597293 & 3.040051 & $1-1.725660$ \\
\hline
\end{tabular}




$\begin{array}{llllll}34 & 6 & 0 & -4.148313 & -1.744219 & -1.547947 \\ 35 & 6 & 0 & -3.837165 & .554746 & -2.324681 \\ 36 & 6 & 0 & -1.893202 & -3.072873 & 3.933946 \\ 37 & 6 & 0 & .646304 & -1.431978 & 4.726993 \\ 38 & 6 & 0 & .186727 & 3.742738 & 1.545089 \\ 39 & 6 & 0 & .956874 & 2.899087 & 2.458349 \\ 40 & 1 & 0 & 1.223396 & .986894 & 3.401814 \\ 41 & 6 & 0 & -1.689902 & 4.033986 & -.042890 \\ 42 & 6 & 0 & 4.026119 & -1.446453 & 1.182988 \\ 43 & 6 & 0 & 4.197529 & -1.824881 & -.217885 \\ 44 & 1 & 0 & 2.720419 & -1.264046 & 2.879198 \\ 45 & 6 & 0 & 3.275886 & -2.722273 & -2.330381 \\ 46 & 1 & 0 & -3.526633 & -2.000370 & 2.150226 \\ 47 & 1 & 0 & -4.412291 & -.497493 & 2.506465 \\ 48 & 1 & 0 & -4.324514 & -1.102811 & .828923 \\ 49 & 1 & 0 & -1.386427 & -4.850506 & 1.087073 \\ 50 & 1 & 0 & -2.462945 & -3.439756 & .923788 \\ 51 & 1 & 0 & -1.848332 & -4.256992 & -.532995 \\ 52 & 1 & 0 & .888545 & -1.482859 & -2.975062 \\ 53 & 1 & 0 & .271965 & -.799382 & -4.496808 \\ 54 & 6 & 0 & 3.637928 & 2.586171 & .156816 \\ 55 & 6 & 0 & 2.540167 & 4.043653 & -1.450516 \\ 56 & 1 & 0 & 2.799047 & .592564 & .371793 \\ 57 & 1 & 0 & .795730 & 3.233667 & -2.448394 \\ 58 & 6 & 0 & -5.540224 & -1.610510 & -1.727299 \\ 59 & 6 & 0 & -5.227498 & .681251 & -2.498140 \\ 60 & 1 & 0 & -3.718930 & -2.689937 & -1.190502 \\ 61 & 1 & 0 & -3.164494 & 1.391413 & -2.560598 \\ 62 & 6 & 0 & .513481 & 5.123063 & 1.354762 \\ 63 & 1 & 0 & 1.804311 & 3.360785 & 2.983051 \\ 64 & 1 & 0 & 4.915565 & -1.111154 & 1.732735 \\ 65 & 6 & 0 & 5.480263 & -1.760914 & -.849735 \\ 66 & 6 & 0 & 4.539086 & -2.650817 & -2.917803 \\ 67 & 1 & 0 & 2.426334 & -3.112550 & -2.905510 \\ 68 & 6 & 0 & -1.346886 & 5.376608 & -.207203 \\ 69 & 1 & 0 & -2.543849 & 3.609758 & -.587642 \\ 70 & 1 & 0 & -2.605312 & -2.531531 & 4.567512 \\ 71 & 1 & 0 & -2.448479 & -3.604815 & 3.156573 \\ 72 & 1 & 0 & -1.379429 & -3.815631 & 4.555871 \\ 73 & 1 & 0 & 1.458745 & -.743873 & 4.477574 \\ 74 & 1 & 0 & .058078 & -.989755 & 5.540042 \\ 75 & 1 & 0 & 1.085789 & -2.367855 & 5.092322 \\ 76 & 6 & 0 & 3.566242 & 3.824331 & -.509204 \\ 79 & 1 & 0 & 4.440721 & 2.397158 & .882188 \\ & 1 & 0 & 2.470437 & 5.007418 & -1.972817 \\ 7 & 0 & -6.082553 & -.399560 & -2.198849\end{array}$




$\begin{array}{llllll}80 & 1 & 0 & -6.201039 & -2.458008 & -1.499673 \\ 81 & 1 & 0 & -5.645706 & 1.626369 & -2.870464 \\ 82 & 6 & 0 & 5.648853 & -2.159741 & -2.175028 \\ 83 & 1 & 0 & 6.337029 & -1.393464 & -.268552 \\ 84 & 1 & 0 & 4.681470 & -2.976930 & -3.956750 \\ 85 & 6 & 0 & -.238177 & 5.925058 & .496129 \\ 86 & 1 & 0 & 1.370287 & 5.543177 & 1.898975 \\ 87 & 1 & 0 & -1.933161 & 6.015411 & -.881358 \\ 88 & 1 & 0 & 4.304266 & 4.609687 & -.301788 \\ 89 & 1 & 0 & -7.167112 & -.297850 & -2.336185 \\ 90 & 1 & 0 & 6.638782 & -2.104811 & -2.646982 \\ 91 & 1 & 0 & .023091 & 6.982899 & .359599 \\ ----------------------------------------------------19\end{array}$

\section{twist (meso-3)}

absolute energy: -1899.89380562 hartrees imaginary frequencies: 0

\begin{tabular}{|c|c|c|c|c|c|}
\hline \multirow{2}{*}{$\begin{array}{l}\text { Center } \\
\text { Number }\end{array}$} & \multirow{2}{*}{$\begin{array}{l}\text { Atomic } \\
\text { Number }\end{array}$} & \multirow{2}{*}{\multicolumn{2}{|c|}{$\begin{array}{r}\text { Atomic } \\
\text { Type }\end{array}$}} & \multicolumn{2}{|c|}{ Coordinates (Angstroms } \\
\hline & & & & $\mathrm{X}$ & $\mathrm{Z}$ \\
\hline 1 & 40 & 0 & .509847 & -.384224 & -.153133 \\
\hline 2 & 7 & 0 & 1.665385 & -.331427 & -1.868871 \\
\hline 3 & 7 & 0 & -.672558 & 1.111725 & -.986707 \\
\hline 4 & 6 & 0 & -.329532 & -2.519383 & 1.196314 \\
\hline 5 & 6 & 0 & .212115 & -2.963576 & -.071979 \\
\hline 6 & 6 & 0 & -.695908 & -2.574874 & -1.123498 \\
\hline 7 & 6 & 0 & -1.847989 & -1.959779 & -.523307 \\
\hline 8 & 6 & 0 & -1.610344 & -1.890926 & .914271 \\
\hline 9 & 6 & 0 & 1.561107 & -.746509 & 2.273016 \\
\hline 10 & 6 & 0 & 2.711479 & -.652073 & 1.414602 \\
\hline 11 & 6 & 0 & 2.761361 & .679583 & .852579 \\
\hline 12 & 6 & 0 & 1.691771 & 1.456159 & 1.409822 \\
\hline 13 & 6 & 0 & .918002 & .567177 & 2.270110 \\
\hline 14 & 6 & 0 & .860272 & -.140327 & -3.152799 \\
\hline 15 & 6 & 0 & .038899 & 2.038520 & -1.977871 \\
\hline 16 & 6 & 0 & 3.108731 & -.344182 & -2.184525 \\
\hline 17 & 6 & 0 & -2.079434 & 1.518327 & -.829003 \\
\hline 18 & 1 & 0 & -.635961 & -2.951800 & -2.148799 \\
\hline 19 & 6 & 0 & 1.364284 & -3.950842 & -.300398 \\
\hline 20 & 6 & 0 & -2.703725 & -1.496626 & 1.797000 \\
\hline 21 & 6 & 0 & 3.885792 & -1.631175 & 1.315964 \\
\hline 22 & 6 & 0 & -.131407 & 1.135445 & 3.112280 \\
\hline 23 & 6 & 0 & 1.421778 & 2.901351 & 1.377198 \\
\hline 24 & 6 & 0 & -3.177026 & -1.675298 & -1.080221 \\
\hline 25 & 1 & 0 & 3.610941 & 1.076522 & .288955 \\
\hline
\end{tabular}




$\begin{array}{llllll}26 & 14 & 0 & .686177 & -2.388112 & 2.848670 \\ 27 & 1 & 0 & -.030016 & -.790113 & -3.104563 \\ 28 & 1 & 0 & 1.469824 & -.460995 & -4.021672 \\ 29 & 6 & 0 & .389340 & 1.344198 & -3.338227 \\ 30 & 1 & 0 & -.603272 & 2.921032 & -2.164701 \\ 31 & 1 & 0 & .969758 & 2.401623 & -1.509212 \\ 32 & 6 & 0 & 3.828361 & -1.566831 & -2.183975 \\ 33 & 6 & 0 & 3.792642 & .851968 & -2.535441 \\ 34 & 6 & 0 & -2.621262 & 1.747172 & .461450 \\ 35 & 6 & 0 & -2.908951 & 1.745151 & -1.965915 \\ 36 & 6 & 0 & 1.887149 & -3.923628 & 3.210645 \\ 37 & 6 & 0 & -.394261 & -2.218549 & 4.500761 \\ 38 & 6 & 0 & -4.232099 & -1.347141 & -.156523 \\ 39 & 6 & 0 & -3.949855 & -1.256252 & 1.277184 \\ 40 & 1 & 0 & -2.534771 & -1.458737 & 2.879727 \\ 41 & 6 & 0 & -3.479593 & -1.793912 & -2.470965 \\ 42 & 6 & 0 & -.365639 & 2.487833 & 3.097520 \\ 43 & 6 & 0 & .382779 & 3.406281 & 2.237243 \\ 44 & 1 & 0 & -.687944 & .481973 & 3.794245 \\ 45 & 6 & 0 & 2.171551 & 3.816655 & .578363 \\ 46 & 1 & 0 & 1.101240 & -4.929523 & .140392 \\ 47 & 1 & 0 & 1.523199 & -4.091447 & -1.379685 \\ 48 & 1 & 0 & 2.309882 & -3.619218 & .147016 \\ 49 & 1 & 0 & 4.681025 & -1.308982 & 2.013220 \\ 50 & 1 & 0 & 3.589891 & -2.650251 & 1.592125 \\ 51 & 1 & 0 & 4.307512 & -1.644122 & .300815 \\ 52 & 1 & 0 & 1.181994 & 1.933009 & -3.830457 \\ 53 & 1 & 0 & -.491806 & 1.343568 & -4.002207 \\ 54 & 1 & 0 & 3.298334 & -2.496449 & -1.937404 \\ 55 & 6 & 0 & 5.198921 & -1.590023 & -2.511484 \\ 56 & 1 & 0 & 3.246703 & 1.804706 & -2.536647 \\ 57 & 6 & 0 & 5.159892 & .820249 & -2.863536 \\ 58 & 1 & 0 & -1.977178 & 1.617324 & 1.340900 \\ 59 & 6 & 0 & -3.951443 & 2.185372 & .611881 \\ 60 & 1 & 0 & -2.513140 & 1.564144 & -2.973049 \\ 61 & 6 & 0 & -4.232268 & 2.186041 & -1.805227 \\ 62 & 6 & 0 & -5.551932 & -1.137944 & -.666898 \\ 63 & 1 & 0 & -4.785093 & -1.000620 & 1.942821 \\ 64 & 1 & 0 & -1.130943 & 2.920401 & 3.756169 \\ 65 & 6 & 0 & .116959 & 4.811326 & 2.244316 \\ 66 & 6 & 0 & 1.891067 & 5.184394 & .609138 \\ 67 & 1 & 0 & 2.973746 & 3.431296 & -.065123 \\ 70 & 6 & 0 & -4.778379 & -1.587470 & -2.938612 \\ 71 & 1 & 0 & -2.674684 & -2.049381 & -3.172948 \\ & 1 & 0 & 1.299302 & -4.737876 & 3.650981 \\ 69 & 0 & 2.372964 & -4.303650 & 2.307905\end{array}$




$\begin{array}{llllll}72 & 1 & 0 & 2.661194 & -3.633827 & 3.930826 \\ 73 & 1 & 0 & -1.130355 & -1.411211 & 4.452322 \\ 74 & 1 & 0 & -.926148 & -3.159475 & 4.685327 \\ 75 & 1 & 0 & .270249 & -2.028431 & 5.352144 \\ 76 & 1 & 0 & 5.742392 & -2.544665 & -2.506424 \\ 77 & 6 & 0 & 5.868304 & -.398791 & -2.850792 \\ 78 & 1 & 0 & 5.676923 & 1.753025 & -3.127084 \\ 79 & 1 & 0 & -4.348752 & 2.367778 & 1.619445 \\ 80 & 6 & 0 & -4.761085 & 2.409205 & -.516807 \\ 81 & 1 & 0 & -4.858228 & 2.353004 & -2.692237 \\ 82 & 6 & 0 & .853776 & 5.684716 & 1.442838 \\ 83 & 1 & 0 & -.680003 & 5.195816 & 2.895421 \\ 84 & 1 & 0 & 2.471923 & 5.878602 & -.012943 \\ 85 & 6 & 0 & -5.821627 & -1.256322 & -2.030782 \\ 86 & 1 & 0 & -6.356541 & -.885039 & .036922 \\ 87 & 1 & 0 & -4.998527 & -1.681191 & -4.010430 \\ 88 & 1 & 0 & 6.935988 & -.418506 & -3.105662 \\ 89 & 1 & 0 & -5.794770 & 2.758363 & -.397426 \\ 90 & 1 & 0 & .638067 & 6.761316 & 1.457165 \\ 91 & 1 & 0 & -6.840549 & -1.095672 & -2.407431 \\ -----------------------------------------------------------\end{array}$

\section{$\mathrm{Cp}_{2} \mathrm{Zr}\left\{\mathrm{PhN}\left(\mathrm{CH}_{2}\right)_{3} \mathrm{NPh}\right\}(5)$ \\ boat (5)}

absolute energy: $-\mathbf{1 1 2 4 . 2 9 1 4 5 7 9 9}$ hartrees imaginary frequencies: 0

\begin{tabular}{|c|c|c|c|c|c|}
\hline \multirow{2}{*}{$\begin{array}{l}\text { Center } \\
\text { Number }\end{array}$} & \multirow{2}{*}{\multicolumn{2}{|c|}{$\begin{array}{l}\text { Atomic } \\
\text { Number }\end{array}$}} & \multirow{2}{*}{$\begin{array}{l}\text { Atomic } \\
\text { Type }\end{array}$} & \multicolumn{2}{|c|}{ Coordinates (Angstroms } \\
\hline & & & & $\mathrm{X}$ & $\mathrm{Z}$ \\
\hline 1 & 40 & 0 & -.035558 & .726706 & -.092013 \\
\hline 2 & 7 & 0 & 1.532288 & -.237243 & .855479 \\
\hline 3 & 7 & 0 & -1.394136 & -.609623 & .800602 \\
\hline 4 & 6 & 0 & .528104 & 1.375826 & -2.590545 \\
\hline 5 & 6 & 0 & -.830219 & .904481 & -2.570400 \\
\hline 6 & 6 & 0 & -.804234 & -.504108 & -2.296130 \\
\hline 7 & 6 & 0 & .557223 & -.892051 & -2.104795 \\
\hline 8 & 6 & 0 & 1.382656 & .271158 & -2.295575 \\
\hline 9 & 6 & 0 & .299682 & 2.578639 & 1.801342 \\
\hline 10 & 6 & 0 & .843936 & 3.182092 & .610146 \\
\hline 11 & 6 & 0 & -.238713 & 3.443882 & -.278200 \\
\hline 12 & 6 & 0 & -1.451910 & 2.977467 & .338838 \\
\hline 13 & 6 & 0 & -1.115724 & 2.482755 & 1.640501 \\
\hline 14 & 6 & 0 & -.948193 & -1.431911 & 2.000725 \\
\hline 15 & 6 & 0 & 1.487862 & -.526979 & 2.354099 \\
\hline
\end{tabular}




$\begin{array}{cccccc}16 & 6 & 0 & 2.807860 & -.777238 & .330132 \\ 17 & 6 & 0 & -2.711676 & -1.020267 & .333810 \\ 18 & 1 & 0 & .920203 & -1.917831 & -1.983272 \\ 19 & 1 & 0 & -.180448 & 4.019017 & -1.207860 \\ 20 & 1 & 0 & -.485402 & -2.383885 & 1.668095 \\ 21 & 1 & 0 & 2.033073 & .264331 & 2.908859 \\ 22 & 1 & 0 & 2.029501 & -1.481881 & 2.509441 \\ 23 & 1 & 0 & -1.849647 & -1.678302 & 2.598210 \\ 24 & 6 & 0 & .045184 & -.676480 & 2.934329 \\ 25 & 1 & 0 & 1.875080 & 3.529126 & .486181 \\ 26 & 1 & 0 & -1.706841 & 1.475273 & -2.895339 \\ 27 & 1 & 0 & 2.474893 & .273207 & -2.360151 \\ 28 & 1 & 0 & -1.833581 & 2.185069 & 2.411649 \\ 29 & 6 & 0 & 3.883646 & .094866 & .027230 \\ 30 & 6 & 0 & 2.984533 & -2.176917 & .164356 \\ 31 & 6 & 0 & -3.160835 & -2.373495 & .425054 \\ 32 & 6 & 0 & -3.594608 & -.066699 & -.249642 \\ 33 & 1 & 0 & .851741 & 2.416570 & 2.731990 \\ 34 & 1 & 0 & -2.463803 & 3.185707 & -.022091 \\ 35 & 1 & 0 & -1.665386 & -1.178927 & -2.333874 \\ 36 & 1 & 0 & .856172 & 2.365095 & -2.923295 \\ 37 & 1 & 0 & .127844 & -1.244682 & 3.877985 \\ 38 & 1 & 0 & -.378947 & .308305 & 3.183562 \\ 39 & 1 & 0 & 3.750461 & 1.176117 & .172335 \\ 40 & 6 & 0 & 5.103534 & -.422359 & -.452094 \\ 41 & 1 & 0 & 2.151878 & -2.854653 & .399052 \\ 42 & 6 & 0 & 4.205814 & -2.685078 & -.312840 \\ 43 & 1 & 0 & -2.505617 & -3.140736 & .852188 \\ 44 & 6 & 0 & -4.429759 & -2.740789 & -.052003 \\ 45 & 1 & 0 & -3.289126 & .986492 & -.285101 \\ 46 & 6 & 0 & -4.857304 & -.447916 & -.733364 \\ 47 & 1 & 0 & 5.928825 & .263082 & -.688039 \\ 48 & 6 & 0 & 5.267626 & -1.810448 & -.623959 \\ 49 & 1 & 0 & 4.328602 & -3.768376 & -.447568 \\ 50 & 1 & 0 & -4.750532 & -3.788552 & .027458 \\ 51 & 6 & 0 & -5.285774 & -1.786588 & -.636868 \\ 52 & 1 & 0 & -5.518981 & .311527 & -1.171569 \\ 53 & 1 & 0 & 6.218670 & -2.211029 & -.998757 \\ 54 & 1 & 0 & -6.275635 & -2.081313 & -1.007027 \\ ---------------------------------------------------------- \\ & & & & & \end{array}$

\section{chair (5) \\ absolute energy: $-\mathbf{1 1 2 4 . 2 9 2 9 7 0 2 3}$ hartrees imaginary frequencies: 1 (-51.2495)}

Center Atomic Atomic Coordinates (Angstroms) 


\begin{tabular}{|c|c|c|c|c|c|}
\hline Number & & Number & Type & $X$ & $\mathrm{Z}$ \\
\hline 1 & 40 & 0 & .000079 & .783818 & -.074931 \\
\hline 2 & 7 & 0 & 1.515951 & -.338989 & .819379 \\
\hline 3 & 7 & 0 & -1.514910 & -.340761 & .819488 \\
\hline 4 & 6 & 0 & .708167 & 1.325959 & -2.530047 \\
\hline 5 & 6 & 0 & -.729590 & 1.309625 & -2.528367 \\
\hline 6 & 6 & 0 & -1.153792 & -.039341 & -2.307674 \\
\hline 7 & 6 & 0 & .014463 & -.855954 & -2.152013 \\
\hline 8 & 6 & 0 & 1.163604 & -.013062 & -2.310426 \\
\hline 9 & 6 & 0 & 1.158694 & 2.783431 & 1.336391 \\
\hline 10 & 6 & 0 & .719677 & 3.354601 & .107290 \\
\hline 11 & 6 & 0 & -.727057 & 3.352644 & .108016 \\
\hline 12 & 6 & 0 & -1.163239 & 2.780391 & 11.337605 \\
\hline 13 & 6 & 0 & -.001369 & 2.391322 & 2.086055 \\
\hline 14 & 6 & 0 & -1.304596 & -.778415 & 2.266613 \\
\hline 15 & 6 & 0 & 1.306586 & -.777199 & 2.266554 \\
\hline 16 & 6 & 0 & 2.775851 & -.877175 & .296122 \\
\hline 17 & 6 & 0 & -2.774897 & -.878659 & .296733 \\
\hline 18 & 1 & 0 & .026580 & -1.949014 & -2.082672 \\
\hline 19 & 1 & 0 & -1.361623 & 3.877011 & $1 \quad-.613504$ \\
\hline 20 & 1 & 0 & -2.173499 & -1.395097 & $\begin{array}{ll}7 & 2.567694\end{array}$ \\
\hline 21 & 1 & 0 & 1.289629 & .116542 & 2.919643 \\
\hline 22 & 1 & 0 & 2.176126 & -1.393313 & 2.567047 \\
\hline 23 & 1 & 0 & -1.288159 & .115536 & 2.919430 \\
\hline 24 & 6 & 0 & .001368 & -1.599053 & 2.496441 \\
\hline 25 & 1 & 0 & 1.352119 & 3.880823 & $\begin{array}{ll}3 & -.614718\end{array}$ \\
\hline 26 & 1 & 0 & -1.376789 & 2.142900 & $\begin{array}{ll}0 & -2.820169\end{array}$ \\
\hline 27 & 1 & 0 & 2.200269 & -.354109 & -2.381367 \\
\hline 28 & 1 & 0 & -.000237 & 2.080953 & 3.135027 \\
\hline 29 & 6 & 0 & 3.754585 & -.007184 & -.255870 \\
\hline 30 & 6 & 0 & 3.057480 & -2.273528 & .342952 \\
\hline 31 & 6 & 0 & -3.059600 & -2.274269 & .348616 \\
\hline 32 & 6 & 0 & -3.750733 & -.008995 & -.261005 \\
\hline 33 & 1 & 0 & 2.192624 & 2.749994 & $4 \quad 1.695885$ \\
\hline 34 & 1 & 0 & -2.196714 & 2.743872 & 1.698116 \\
\hline 35 & 1 & 0 & -2.182763 & -.403499 & -2.375615 \\
\hline 36 & 1 & 0 & 1.335739 & 2.173386 & $5 \quad-2.823860$ \\
\hline 37 & 1 & 0 & 3.556376 & 1.073671 & $1-.269564$ \\
\hline 38 & 6 & 0 & 4.966084 & -.518030 & -.759440 \\
\hline 39 & 1 & 0 & 2.306515 & -2.962201 & .751505 \\
\hline 40 & 6 & 0 & 4.272830 & -2.772698 & -.154064 \\
\hline 41 & 1 & 0 & -2.310854 & -2.963011 & .761066 \\
\hline 42 & 6 & 0 & -4.274997 & -2.772888 & -.148782 \\
\hline 43 & 1 & 0 & -3.550081 & 1.071349 & $9 \quad-.279028$ \\
\hline 44 & 6 & 0 & -4.962215 & -.519360 & -.764988 \\
\hline
\end{tabular}




$\begin{array}{llllll}45 & 1 & 0 & .001574 & -1.952191 & 3.543891 \\ 46 & 1 & 0 & .001749 & -2.482082 & 1.834189 \\ 47 & 1 & 0 & 5.710879 & .170688 & -1.180721 \\ 48 & 6 & 0 & 5.231631 & -1.899932 & -.709424 \\ 49 & 1 & 0 & 4.470391 & -3.852712 & -.117088 \\ 50 & 1 & 0 & -4.474834 & -3.852341 & -.107834 \\ 51 & 6 & 0 & -5.230866 & -1.900471 & -.709706 \\ 52 & 1 & 0 & -5.704618 & .169182 & -1.190753 \\ 53 & 1 & 0 & 6.178753 & -2.295600 & -1.098164 \\ 54 & 1 & 0 & -6.178019 & -2.295758 & -1.098738 \\ -\end{array}$

\section{twist (5)}

absolute energy: $-\mathbf{1 1 2 4 . 2 9 7 1 8 9 8 3}$ hartrees imaginary frequencies: 0

\begin{tabular}{|c|c|c|c|c|c|}
\hline \multirow{2}{*}{$\begin{array}{l}\text { Center } \\
\text { Number }\end{array}$} & \multirow{2}{*}{$\begin{array}{l}\text { Atomic } \\
\text { Number }\end{array}$} & \multirow{2}{*}{\multicolumn{2}{|c|}{$\begin{array}{l}\text { Atomic } \\
\text { Type }\end{array}$}} & \multicolumn{2}{|c|}{ Coordinates (Angstrom } \\
\hline & & & & $\mathrm{X}$ & $\mathrm{Z}$ \\
\hline 1 & 6 & 0 & -.769038 & 2.074252 & 1.060302 \\
\hline 2 & 1 & 0 & -.064141 & 1.788939 & 1.860911 \\
\hline 3 & 1 & 0 & -1.575548 & 2.678444 & 1.521346 \\
\hline 4 & 6 & 0 & -.000428 & 2.938770 & -.000334 \\
\hline 5 & 7 & 0 & -1.369586 & .800142 & .474998 \\
\hline 6 & 1 & 0 & -.708005 & 3.590014 & -.540938 \\
\hline 7 & 1 & 0 & .706882 & 3.590450 & .540101 \\
\hline 8 & 6 & 0 & .76 & 270 & -1.060731 \\
\hline 9 & 6 & 0 & -2.825727 & .910921 & .340023 \\
\hline 10 & 40 & 0 & -.000112 & -.699873 & .000244 \\
\hline 11 & 1 & 0 & 1.575206 & 2.678538 & -1.521453 \\
\hline 12 & 7 & 0 & 1.369075 & .800219 & -.475266 \\
\hline 13 & 1 & 0 & .063998 & 1.788953 & -1.861622 \\
\hline 14 & 6 & 0 & -3.670289 & -.082315 & .904818 \\
\hline 15 & 6 & 0 & -3.429405 & 2.010732 & -.334850 \\
\hline 16 & 1 & 0 & -3.212992 & -.912058 & 1.461708 \\
\hline 17 & 6 & 0 & -5.069279 & .007282 & .777323 \\
\hline 18 & 1 & 0 & -2.795912 & 2.786469 & -.782991 \\
\hline 19 & 6 & 0 & -4.827021 & 2.095896 & -.450220 \\
\hline 20 & 6 & 0 & 2.825239 & .911014 & -.340406 \\
\hline 21 & 6 & 0 & 1.060962 & -2.805468 & 1.291009 \\
\hline 22 & 6 & 0 & -.156765 & -2.436607 & 1.952479 \\
\hline 23 & 6 & 0 & .052626 & -1.157504 & 2.584209 \\
\hline 24 & 6 & 0 & 1.372575 & -.724259 & 2.269118 \\
\hline 25 & 6 & 0 & 1.994930 & -1.739335 & 1.455252 \\
\hline 26 & 6 & 0 & -.045947 & -1.164887 & -2.582365 \\
\hline 27 & 6 & 0 & .154535 & -2.443185 & -1.946192 \\
\hline
\end{tabular}




$\begin{array}{cccccc}28 & 6 & 0 & -1.067549 & -2.803955 & -1.288255 \\ 29 & 6 & 0 & -1.995499 & -1.733699 & -1.459379 \\ 30 & 6 & 0 & -1.364873 & -.723961 & -2.273470 \\ 31 & 1 & 0 & -5.704821 & -.770208 & 1.222429 \\ 32 & 6 & 0 & -5.653731 & 1.094741 & .100015 \\ 33 & 1 & 0 & -5.275209 & 2.947147 & -.980640 \\ 34 & 6 & 0 & 3.669893 & -.081973 & -.905474 \\ 35 & 6 & 0 & 3.428811 & 2.010758 & .334679 \\ 36 & 1 & 0 & 1.880441 & .151045 & 2.687387 \\ 37 & 1 & 0 & -1.285211 & -3.776351 & -.834563 \\ 38 & 1 & 0 & 1.007672 & -3.108188 & -2.116898 \\ 39 & 1 & 0 & -1.012669 & -3.096755 & 2.128221 \\ 40 & 1 & 0 & 3.053212 & -1.767080 & 1.179688 \\ 41 & 1 & 0 & -1.866791 & .152534 & -2.696391 \\ 42 & 1 & 0 & .644942 & -.687840 & -3.285219 \\ 43 & 1 & 0 & -3.054887 & -1.755135 & -1.187522 \\ 44 & 1 & 0 & -.633232 & -.674927 & 3.288208 \\ 45 & 1 & 0 & 1.271953 & -3.780278 & .839372 \\ 46 & 1 & 0 & -6.744928 & 1.165974 & .005358 \\ 47 & 1 & 0 & 3.212716 & -.911614 & -1.462600 \\ 48 & 6 & 0 & 5.068882 & .007813 & -.778042 \\ 49 & 1 & 0 & 2.795212 & 2.786316 & .782994 \\ 50 & 6 & 0 & 4.826418 & 2.096112 & .449996 \\ 51 & 1 & 0 & 5.704523 & -.769462 & -1.223386 \\ 52 & 6 & 0 & 5.653228 & 1.095190 & -.100513 \\ 53 & 1 & 0 & 5.274513 & 2.947311 & .980576 \\ 54 & 1 & 0 & 6.744423 & 1.166544 & -.005916 \\ & 1 & & & & \end{array}$

\section{rac-(MBSBI)Zr $\left\{\mathrm{PhN}\left(\mathrm{CH}_{2}\right)_{2} \mathrm{NPh}\right\}$ (7) absolute energy: imaginary frequencies: 0}

\begin{tabular}{|c|c|c|c|c|c|}
\hline \multirow{2}{*}{$\begin{array}{l}\text { Center } \\
\text { Number }\end{array}$} & \multirow{2}{*}{$\begin{array}{l}\text { Atomic } \\
\text { Number }\end{array}$} & \multirow{2}{*}{\multicolumn{2}{|c|}{$\begin{array}{l}\text { Atomic } \\
\text { Type }\end{array}$}} & \multicolumn{2}{|c|}{ Coordinates (Angstrom } \\
\hline & & & & $\mathrm{X}$ & $\mathrm{Z}$ \\
\hline 1 & 40 & 0 & -0.09 & 181161 & 40934 \\
\hline 2 & 7 & 0 & -1.108210 & -1.608602 & 0.080665 \\
\hline 3 & 7 & 0 & 1.06 & -0.756342 & 1.533052 \\
\hline 4 & 6 & 0 & 0.49 & -2.114093 & 1.925798 \\
\hline 5 & 6 & 0 & -0.996704 & -2.175339 & 1.493414 \\
\hline 6 & 6 & 0 & -1.743609 & -2.547994 & -0.851481 \\
\hline 7 & 6 & 0 & 2.327554 & -0.477922 & 2.204837 \\
\hline 8 & 1 & 0 & 1.062821 & -2.931586 & 1.431293 \\
\hline 9 & 1 & 0 & 0.574075 & -2.244811 & 3.025453 \\
\hline 10 & 1 & 0 & -1.366415 & -3.217960 & 1.50692 \\
\hline
\end{tabular}




$\begin{array}{rrrrrr}11 & 1 & 0 & -1.613133 & -1.576982 & 2.190580 \\ 12 & 6 & 0 & -1.225904 & -3.862288 & -1.025181 \\ 13 & 6 & 0 & -2.889800 & -2.156334 & -1.590042 \\ 14 & 6 & 0 & 2.812767 & 0.858376 & 2.266663 \\ 15 & 6 & 0 & 3.118999 & -1.498008 & 2.815953 \\ 16 & 1 & 0 & -0.330442 & -4.169929 & -0.468709 \\ 17 & 6 & 0 & -1.841014 & -4.752205 & -1.920482 \\ 18 & 1 & 0 & -3.309646 & -1.153375 & -1.428769 \\ 19 & 6 & 0 & -3.496017 & -3.056999 & -2.489133 \\ 20 & 1 & 0 & 2.216220 & 1.659113 & 1.817614 \\ 21 & 6 & 0 & 4.021432 & 1.166749 & 2.907290 \\ 22 & 1 & 0 & 2.802966 & -2.544601 & 2.779343 \\ 23 & 6 & 0 & 4.327850 & -1.177215 & 3.457413 \\ 24 & 1 & 0 & -1.428259 & -5.762344 & -2.051110 \\ 25 & 6 & 0 & -2.975305 & -4.353817 & -2.657586 \\ 26 & 1 & 0 & -4.384972 & -2.742626 & -3.050223 \\ 27 & 1 & 0 & 4.361938 & 2.210125 & 2.945545 \\ 28 & 6 & 0 & 4.790511 & 0.150837 & 3.511600 \\ 29 & 1 & 0 & 4.917710 & -1.982491 & 3.916838 \\ 30 & 1 & 0 & -3.452471 & -5.052261 & -3.357170 \\ 31 & 1 & 0 & 5.734042 & 0.391542 & 4.016690 \\ 32 & 6 & 0 & -1.078864 & 2.710134 & -0.102887 \\ 33 & 6 & 0 & -0.292481 & 2.720335 & 1.111291 \\ 34 & 6 & 0 & -2.207641 & 1.826791 & 0.127815 \\ 35 & 14 & 0 & -0.432928 & 3.245874 & -1.855370 \\ 36 & 6 & 0 & -0.905389 & 1.833825 & 2.065832 \\ 37 & 6 & 0 & -2.096946 & 1.283278 & 1.482547 \\ 38 & 6 & 0 & 0.413665 & 1.519802 & -2.156464 \\ 39 & 6 & 0 & 0.797037 & 3.735609 & 1.481910 \\ 40 & 6 & 0 & -3.427385 & 1.585474 & -0.635081 \\ 41 & 6 & 0 & 0.810288 & 4.788616 & -1.865770 \\ 42 & 6 & 0 & -1.837238 & 3.725115 & -3.169739 \\ 43 & 6 & 0 & -0.239409 & 0.331042 & -2.653118 \\ 44 & 6 & 0 & 1.700779 & 1.099272 & -1.609843 \\ 45 & 1 & 0 & -0.612275 & 1.757210 & 3.117522 \\ 46 & 6 & 0 & -3.209465 & 0.527755 & 2.079958 \\ 47 & 6 & 0 & -4.460052 & 0.872047 & -0.077074 \\ 48 & 1 & 0 & -3.536404 & 2.035220 & -1.629126 \\ 49 & 1 & 0 & 1.690023 & 3.668775 & 0.845224 \\ 50 & 1 & 0 & 0.390760 & 4.757962 & 1.388058 \\ 51 & 1 & 0 & 1.108415 & 3.585228 & 2.527360 \\ 52 & 1 & 0 & 0.239370 & 5.716663 & -1.744437 \\ 53 & 1 & 0 & 1.555545 & 4.739202 & -1.066939 \\ 56 & 1 & 0 & 1.333714 & 4.832933 & -2.829546 \\ & 1 & 0 & -1.370706 & 3.972187 & -4.130722 \\ 54 & 0 & -2.558794 & 2.919096 & -3.334999\end{array}$




\begin{tabular}{|c|c|c|c|c|c|}
\hline 57 & 1 & 0 & -2.380915 & 4.609316 & -2.816032 \\
\hline 58 & 6 & 0 & 0.593924 & -0.806441 & -2.368310 \\
\hline 59 & 6 & 0 & 1.810022 & -0.349605 & -1.749781 \\
\hline 60 & 6 & 0 & -1.475439 & 0.259604 & -3.560066 \\
\hline 61 & 6 & 0 & 2.866947 & 1.857352 & -1.165786 \\
\hline 62 & 6 & 0 & -4.388692 & 0.320626 & 1.277179 \\
\hline 63 & 6 & 0 & -3.185495 & 0.035220 & 3.420371 \\
\hline 64 & 1 & 0 & -5.391593 & 0.721087 & -0.637328 \\
\hline 65 & 6 & 0 & 3.073191 & -1.044845 & -1.463110 \\
\hline 66 & 1 & 0 & 0.409311 & -1.820179 & -2.739625 \\
\hline 67 & 6 & 0 & 4.050500 & 1.207095 & -0.914892 \\
\hline 68 & 1 & 0 & 2.807504 & 2.951238 & -1.101650 \\
\hline 69 & 1 & 0 & -1.716037 & -0.791280 & -3.779017 \\
\hline 70 & 1 & 0 & -2.359481 & 0.724447 & -3.108546 \\
\hline 71 & 1 & 0 & -1.260456 & 0.777181 & -4.512304 \\
\hline 72 & 6 & 0 & -5.488017 & -0.398808 & 1.842504 \\
\hline 73 & 6 & 0 & -4.278340 & -0.657948 & 3.946720 \\
\hline 74 & 1 & 0 & -2.292973 & 0.208083 & 4.036183 \\
\hline 75 & 6 & 0 & 4.194032 & -0.243844 & -1.047414 \\
\hline 76 & 6 & 0 & 3.238108 & -2.453876 & -1.617867 \\
\hline 77 & 1 & 0 & 4.939839 & 1.779835 & -0.617496 \\
\hline 78 & 6 & 0 & -5.433419 & -0.883903 & 3.150305 \\
\hline 79 & 1 & 0 & -6.384735 & -0.561947 & 1.229109 \\
\hline 80 & 1 & 0 & -4.244950 & -1.032967 & 4.978010 \\
\hline 81 & 6 & 0 & 5.445132 & -0.890908 & -0.788499 \\
\hline 82 & 6 & 0 & 4.471335 & -3.056272 & -1.359506 \\
\hline 83 & 1 & 0 & 2.382106 & -3.060479 & -1.941264 \\
\hline 84 & 1 & 0 & -6.285791 & -1.435033 & 3.569490 \\
\hline 85 & 6 & 0 & 5.580200 & -2.271335 & -0.939283 \\
\hline 86 & 1 & 0 & 6.299984 & -0.280945 & -0.465723 \\
\hline 87 & 1 & 0 & 4.587843 & -4.141806 & -1.479390 \\
\hline 88 & 1 & 0 & 6.543341 & -2.756674 & -0.735360 \\
\hline
\end{tabular}

\section{meso-(MBSBI)Zr $\left\{\mathrm{PhN}\left(\mathrm{CH}_{2}\right)_{2} \mathrm{NPh}\right\}$ (7) absolute energy: imaginary frequencies: 0}

\begin{tabular}{lrrrrr}
\multicolumn{2}{c}{ Center } & \multicolumn{2}{c}{ Atomic } & Atomic & \multicolumn{2}{c}{ Coordinates (Angstroms) } \\
Number & Number & Type & \multicolumn{1}{c}{ X } & Y & Z \\
-1 & 40 & 0 & 0.552916 & -0.410159 & -0.209235 \\
2 & 7 & 0 & -0.722723 & 0.926606 & -1.216913 \\
3 & 7 & 0 & 1.793871 & -0.032468 & -1.806381 \\
4 & 6 & 0 & 0.895198 & 0.251217 & -3.007194 \\
5 & 6 & 0 & -0.173321 & 1.304910 & -2.584769
\end{tabular}




\begin{tabular}{ccrrrr}
6 & 6 & 0 & -1.904718 & 1.711856 & -0.869353 \\
7 & 6 & 0 & 3.211975 & 0.201980 & -2.097529 \\
8 & 1 & 0 & 1.504877 & 0.628659 & -3.851776 \\
9 & 1 & 0 & 0.389411 & -0.681076 & -3.326442 \\
10 & 1 & 0 & 0.299424 & 2.308920 & -2.553165 \\
11 & 1 & 0 & -0.998465 & 1.323067 & -3.327698 \\
12 & 6 & 0 & -2.247743 & 2.925878 & -1.536845 \\
13 & 6 & 0 & -2.762355 & 1.264779 & 0.174707 \\
14 & 6 & 0 & 4.160017 & -0.834097 & -1.911743 \\
15 & 6 & 0 & 3.655932 & 1.464933 & -2.582597 \\
16 & 6 & 0 & -0.082694 & -2.775557 & 0.947664 \\
17 & 1 & 0 & -1.616198 & 3.319639 & -2.338443 \\
18 & 6 & 0 & -3.396051 & 3.647509 & -1.165518 \\
19 & 1 & 0 & -2.539233 & 0.317764 & 0.682893 \\
20 & 6 & 0 & -3.903572 & 1.994893 & 0.538368 \\
21 & 1 & 0 & 3.813715 & -1.818674 & -1.570317 \\
22 & 6 & 0 & 5.524035 & -0.610305 & -2.188920 \\
23 & 1 & 0 & 2.930504 & 2.277663 & -2.717267 \\
24 & 6 & 0 & 5.016551 & 1.676151 & -2.862483 \\
25 & 6 & 0 & 0.605541 & -3.019264 & -0.296542 \\
26 & 6 & 0 & -1.425722 & -2.317462 & 0.607701 \\
27 & 14 & 0 & 0.776157 & -2.559567 & 2.677535 \\
28 & 1 & 0 & -3.636416 & 4.579254 & -1.695987 \\
29 & 6 & 0 & -4.231528 & 3.192222 & -0.129614 \\
30 & 1 & 0 & -4.551318 & 1.616897 & 1.341385 \\
31 & 1 & 0 & 6.247092 & -1.424153 & -2.044243 \\
32 & 6 & 0 & 5.955555 & 0.642439 & -2.663785 \\
33 & 1 & 0 & 5.349205 & 2.657272 & -3.228318 \\
34 & 6 & 0 & -0.270868 & -2.652140 & -1.392116 \\
35 & 6 & 0 & -1.547245 & -2.279124 & -0.840035 \\
36 & 6 & 0 & 1.398543 & -0.762322 & 2.240563 \\
37 & 6 & 0 & 1.905169 & -3.803427 & -0.512560 \\
38 & 6 & 0 & -2.620774 & -2.160480 & 1.426566 \\
39 & 6 & 0 & 2.208142 & -3.872603 & 3.071534 \\
40 & 6 & 0 & -0.425845 & -2.654048 & 4.248683 \\
41 & 1 & 0 & -5.127609 & 3.758716 & 0.151362 \\
42 & 1 & 0 & 7.017087 & 0.814310 & -2.882950 \\
43 & 6 & 0 & 2.615942 & -0.430634 & 1.542228 \\
44 & 6 & 0 & 0.568108 & 0.443230 & 2.240495 \\
45 & 1 & 0 & -0.090424 & -2.940608 & -2.432199 \\
46 & 6 & 0 & -2.847656 & -2.048675 & -1.478740 \\
50 & 6 & 0 & -3.844981 & -1.994135 & 0.829547 \\
51 & 1 & 0 & -2.539504 & -2.234534 & 2.518885 \\
& 1 & 0 & 2.186493 & -3.772894 & -1.575154 \\
49 & 0 & 2.745549 & -3.414351 & 0.076384 \\
\hline & 1 & 1.748587 & -4.861286 & -0.225586
\end{tabular}




\begin{tabular}{|c|c|c|c|c|c|}
\hline 52 & 1 & 0 & 1.750650 & -4.787973 & 3.464188 \\
\hline 53 & 1 & 0 & 2.794631 & -4.136673 & 2.187494 \\
\hline 54 & 1 & 0 & 2.886136 & -3.475279 & 3.836272 \\
\hline 55 & 1 & 0 & 0.160205 & -2.492018 & 5.162927 \\
\hline 56 & 1 & 0 & -1.229379 & -1.913955 & 4.223201 \\
\hline 57 & 1 & 0 & -0.876600 & -3.652779 & 4.305230 \\
\hline 58 & 6 & 0 & 2.515776 & 0.919375 & 1.051327 \\
\hline 59 & 6 & 0 & 1.275204 & 1.488588 & 1.512381 \\
\hline 60 & 6 & 0 & 3.933430 & -1.214698 & 1.543222 \\
\hline 61 & 6 & 0 & -0.657959 & 0.785664 & 2.956990 \\
\hline 62 & 6 & 0 & -3.999896 & -1.922237 & -0.623157 \\
\hline 63 & 6 & 0 & -3.020645 & -1.994268 & -2.895508 \\
\hline 64 & 1 & 0 & -4.753074 & -1.910729 & 1.441586 \\
\hline 65 & 6 & 0 & 0.787929 & 2.875824 & 1.510812 \\
\hline 66 & 1 & 0 & 3.342138 & 1.476139 & 0.599634 \\
\hline 67 & 6 & 0 & -1.098922 & 2.086719 & 2.968768 \\
\hline 68 & 1 & 0 & -1.184788 & 0.013409 & 3.529077 \\
\hline 69 & 1 & 0 & 4.472997 & -1.007435 & 2.484877 \\
\hline 70 & 1 & 0 & 3.775954 & -2.297853 & 1.479276 \\
\hline 71 & 1 & 0 & 4.570303 & -0.900886 & 0.704777 \\
\hline 72 & 6 & 0 & -5.285052 & -1.733388 & -1.224515 \\
\hline 73 & 6 & 0 & -4.287306 & -1.809088 & -3.452117 \\
\hline 74 & 1 & 0 & -2.140288 & -2.096194 & -3.545871 \\
\hline 75 & 6 & 0 & -0.405637 & 3.161842 & 2.258993 \\
\hline 76 & 6 & 0 & 1.469192 & 3.939668 & 0.844099 \\
\hline 77 & 1 & 0 & -2.001344 & 2.352984 & 3.537812 \\
\hline 78 & 6 & 0 & -5.427159 & -1.674457 & -2.610971 \\
\hline 79 & 1 & 0 & -6.164586 & -1.633448 & -0.572979 \\
\hline 80 & 1 & 0 & -4.407321 & -1.761844 & -4.543024 \\
\hline 81 & 6 & 0 & -0.885187 & 4.509610 & 2.302817 \\
\hline 82 & 6 & 0 & 0.977283 & 5.245195 & 0.906060 \\
\hline 83 & 1 & 0 & 2.381917 & 3.716120 & 0.275372 \\
\hline 84 & 1 & 0 & -6.419393 & -1.526965 & -3.058367 \\
\hline 85 & 6 & 0 & -0.208154 & 5.530856 & 1.636840 \\
\hline 86 & 1 & 0 & -1.800893 & 4.727173 & 2.869470 \\
\hline 87 & 1 & 0 & 1.505967 & 6.055684 & 0.386353 \\
\hline 88 & 1 & 0 & -0.588077 & 6.561658 & 1.675781 \\
\hline
\end{tabular}

\section{rac-(SBI)Zr(NMe $)_{2}(8)$ absolute energy: $-\mathbf{1 0 9 2 . 5 1 8 2 2 0 4 7}$ hartrees imaginary frequencies: 0}

\begin{tabular}{|c|c|c|c|}
\hline Center & Atomic & Atomic & Coordinates (Angstroms) \\
\hline Number & Number & Type & $\mathrm{X}$ \\
\hline
\end{tabular}




$\begin{array}{cccccc}1 & 40 & 0 & .000179 & -.617233 & .000110 \\ 2 & 7 & 0 & -1.036848 & -1.995582 & -1.140014 \\ 3 & 7 & 0 & 1.037496 & -1.995083 & 1.140556 \\ 4 & 6 & 0 & -1.314204 & -.244615 & 2.337611 \\ 5 & 6 & 0 & -.525549 & .930825 & 2.099221 \\ 6 & 6 & 0 & -1.021136 & 1.630511 & .951439 \\ 7 & 6 & 0 & -2.186966 & .890683 & .479661 \\ 8 & 6 & 0 & -2.381387 & -.260798 & 1.366886 \\ 9 & 6 & 0 & 1.020699 & 1.630790 & -.951431 \\ 10 & 6 & 0 & 2.186726 & .891023 & -.480055 \\ 11 & 6 & 0 & 2.380971 & -.260387 & -1.367414 \\ 12 & 6 & 0 & 1.313535 & -.244171 & -2.337867 \\ 13 & 6 & 0 & .524844 & .931160 & -2.099133 \\ 14 & 6 & 0 & -1.654573 & -3.225391 & -.503428 \\ 15 & 6 & 0 & -1.510202 & -1.980527 & -2.574769 \\ 16 & 6 & 0 & 1.655830 & -3.224730 & .504249 \\ 17 & 6 & 0 & 1.510416 & -1.979734 & 2.575447 \\ 18 & 1 & 0 & .266463 & 1.295327 & 2.764063 \\ 19 & 6 & 0 & -3.568433 & -1.073079 & 1.239746 \\ 20 & 6 & 0 & 3.181851 & 1.170338 & .532078 \\ 21 & 1 & 0 & 1.263470 & -.875217 & -3.230616 \\ 22 & 1 & 0 & -1.264335 & -.875724 & 3.230325 \\ 23 & 1 & 0 & 1.319637 & -4.130429 & 1.048022 \\ 24 & 1 & 0 & 1.349756 & -3.309272 & -.548764 \\ 25 & 1 & 0 & 2.759835 & -3.171718 & .551040 \\ 26 & 1 & 0 & 1.125814 & -1.096114 & 3.100987 \\ 27 & 1 & 0 & 1.158273 & -2.891707 & 3.099087 \\ 28 & 1 & 0 & 2.617524 & -1.959703 & 2.613167 \\ 29 & 1 & 0 & -2.617330 & -1.961044 & -2.612148 \\ 30 & 1 & 0 & -1.126208 & -1.096737 & -3.100468 \\ 31 & 1 & 0 & -1.157772 & -2.892349 & -3.098477 \\ 32 & 1 & 0 & -1.348116 & -3.309784 & .549485 \\ 33 & 1 & 0 & -2.758610 & -3.172726 & -.549845 \\ 34 & 1 & 0 & -1.318282 & -4.131011 & -1.047271 \\ 35 & 14 & 0 & -.000210 & 2.967208 & .000209 \\ 36 & 6 & 0 & 3.568117 & -1.072593 & -1.240665 \\ 37 & 1 & 0 & -.267439 & 1.295620 & -2.763678 \\ 38 & 6 & 0 & -3.181831 & 1.170019 & -.532713 \\ 39 & 6 & 0 & -1.048982 & 4.100909 & -1.234332 \\ 40 & 6 & 0 & 1.048571 & 4.100424 & 1.235177 \\ 41 & 6 & 0 & 4.307213 & .365548 & .616554 \\ 42 & 1 & 0 & 3.065795 & 2.045079 & 1.184794 \\ 43 & 6 & 0 & 4.504605 & -.751089 & -.272344 \\ 46 & 1 & 0 & 3.732386 & -1.907328 & -1.936590 \\ & 6 & 0 & -4.504686 & -.751550 & .271202 \\ & 1 & 0 & -3.732830 & -1.907889 & 1.935548\end{array}$




\begin{tabular}{|c|c|c|c|c|c|}
\hline 47 & 6 & 0 & -4.307123 & .365168 & -.617555 \\
\hline 48 & 1 & 0 & -3.065640 & 2.044803 & -1.185348 \\
\hline 49 & 1 & 0 & 5.424250 & -1.343966 & -.180107 \\
\hline 50 & 1 & 0 & 5.081874 & .589676 & 1.362179 \\
\hline 51 & 1 & 0 & -1.484236 & 3.503931 & -2.043492 \\
\hline 52 & 1 & 0 & -1.860536 & 4.605769 & -.697990 \\
\hline 53 & 1 & 0 & -.402225 & 4.865187 & -1.681019 \\
\hline 54 & 1 & 0 & .401774 & 4.864397 & 1.682327 \\
\hline 55 & 1 & 0 & 1.483992 & 3.503092 & 2.043984 \\
\hline 56 & 1 & 0 & 1.859999 & 4.605660 & 698998 \\
\hline 57 & 1 & 0 & -5.424267 & -1.344479 & .178668 \\
\hline 58 & 1 & 0 & -5.081596 & .589305 & -1.363373 \\
\hline
\end{tabular}

\section{meso-(SBI)Zr( $\left(\mathrm{NMe}_{2}\right)_{2}$ (8)}

absolute energy: -1092.51797273 hartrees imaginary frequencies: 0

\begin{tabular}{|c|c|c|c|c|c|}
\hline \multirow{2}{*}{$\begin{array}{l}\text { Center } \\
\text { Number }\end{array}$} & \multirow{2}{*}{$\begin{array}{l}\text { Atomic } \\
\text { Number }\end{array}$} & \multirow{2}{*}{\multicolumn{2}{|c|}{$\begin{array}{l}\text { Atomic } \\
\text { Type }\end{array}$}} & \multicolumn{2}{|c|}{ Coordinates (Angstroms } \\
\hline & & & & $\mathrm{X}$ & $\mathrm{Z}$ \\
\hline 1 & 40 & 0 & -.763898 & .058131 & -.486572 \\
\hline 2 & 7 & 0 & -2.307206 & -.008915 & -1.863172 \\
\hline 3 & 7 & 0 & -1.643965 & 1.344339 & .859298 \\
\hline 4 & 6 & 0 & 1.007076 & 2.344868 & -.927634 \\
\hline 5 & 6 & 0 & 1.825155 & 1.315795 & -.282769 \\
\hline 6 & 6 & 0 & 1.839600 & .142351 & -1.150597 \\
\hline 7 & 6 & 0 & 1.050076 & .470259 & -2.299572 \\
\hline 8 & 6 & 0 & .469282 & 1.776963 & -2.145873 \\
\hline 9 & 6 & 0 & .653084 & -2.099088 & .035478 \\
\hline 10 & 6 & 0 & .132707 & -1.666734 & 1.336750 \\
\hline 11 & 6 & 0 & -1.310335 & -1.910060 & 1.350299 \\
\hline 12 & 6 & 0 & -1.674509 & -2.407938 & .044264 \\
\hline 13 & 6 & 0 & -.461604 & -2.582879 & -.710511 \\
\hline 14 & 6 & 0 & -2.814059 & 1.302449 & -2.441290 \\
\hline 15 & 6 & 0 & -3.021943 & -1.096931 & -2.630714 \\
\hline 16 & 6 & 0 & -3.138947 & 1.480313 & 1.050933 \\
\hline 17 & 6 & 0 & -.993573 & 2.119783 & 1.982579 \\
\hline 18 & 6 & 0 & 2.579036 & 1.657358 & .898830 \\
\hline 19 & 1 & 0 & -.000555 & 2.348580 & -2.951460 \\
\hline 20 & 6 & 0 & .777124 & -1.226577 & 2.552836 \\
\hline 21 & 1 & 0 & -2.648325 & -2.838427 & $7 \quad-.208168$ \\
\hline 22 & 6 & 0 & .979228 & 3.678603 & -.387909 \\
\hline 23 & 1 & 0 & -3.437804 & 1.075076 & 2.037359 \\
\hline 24 & 1 & 0 & -3.425080 & 2.550868 & 1.007701 \\
\hline 25 & 1 & 0 & -3.678802 & .932623 & .265373 \\
\hline
\end{tabular}




$\begin{array}{cccccc}26 & 1 & 0 & -1.285326 & 3.186844 & 1.917613 \\ 27 & 1 & 0 & -1.323877 & 1.722274 & 2.961754 \\ 28 & 1 & 0 & .102281 & 2.051022 & 1.923081 \\ 29 & 1 & 0 & -2.666712 & -2.087139 & -2.321854 \\ 30 & 1 & 0 & -4.113963 & -1.031892 & -2.452437 \\ 31 & 1 & 0 & -2.837243 & -.974691 & -3.716667 \\ 32 & 1 & 0 & -3.915460 & 1.349717 & -2.331902 \\ 33 & 1 & 0 & -2.373040 & 2.157845 & -1.909602 \\ 34 & 1 & 0 & -2.560467 & 1.375046 & -3.516532 \\ 35 & 14 & 0 & 2.373703 & -1.668723 & -.734835 \\ 36 & 6 & 0 & -2.061884 & -1.734722 & 2.567433 \\ 37 & 1 & 0 & -.401133 & -3.082332 & -1.684552 \\ 38 & 1 & 0 & .977850 & -.135435 & -3.211461 \\ 39 & 6 & 0 & 2.655075 & -2.707677 & -2.391493 \\ 40 & 6 & 0 & 3.932698 & -1.876685 & .465600 \\ 41 & 6 & 0 & 2.536548 & 2.958699 & 1.379399 \\ 42 & 1 & 0 & 3.218098 & .903275 & 1.376106 \\ 43 & 1 & 0 & .388338 & 4.457850 & -.888943 \\ 44 & 6 & 0 & 1.743554 & 3.969734 & .732710 \\ 45 & 6 & 0 & .018568 & -1.073310 & 3.704972 \\ 46 & 1 & 0 & 1.863161 & -1.069323 & 2.570887 \\ 47 & 6 & 0 & -1.396793 & -1.333198 & 3.715813 \\ 48 & 1 & 0 & -3.138565 & -1.955536 & 2.578856 \\ 49 & 1 & 0 & 1.753973 & 4.989961 & 1.138719 \\ 50 & 1 & 0 & 3.129158 & 3.234483 & 2.261603 \\ 51 & 1 & 0 & -1.948848 & -1.219140 & 4.658183 \\ 52 & 1 & 0 & .506680 & -.767100 & 4.640015 \\ 53 & 1 & 0 & 1.800217 & -2.591091 & -3.067078 \\ 54 & 1 & 0 & 3.556002 & -2.362653 & -2.911404 \\ 55 & 1 & 0 & 2.773878 & -3.772943 & -2.163138 \\ 56 & 1 & 0 & 4.839590 & -1.515702 & -.033576 \\ 57 & 1 & 0 & 3.804093 & -1.316324 & 1.397107 \\ 58 & 1 & 0 & 4.073158 & -2.935055 & .714894 \\ -------------------------------------------------------------- \\ \end{array}$

\section{$\operatorname{rac}-(\mathrm{EBI}) \mathrm{Zr}\left(\mathrm{NMe}_{2}\right)_{2}(9)$ absolute energy: $\mathbf{- 1 0 8 7 . 4 0 6 4 8 3 5 4}$ hartrees imaginary frequencies: 0}

\begin{tabular}{|c|c|c|c|c|c|}
\hline \multirow{2}{*}{$\begin{array}{l}\text { Center } \\
\text { Number }\end{array}$} & \multirow{2}{*}{\multicolumn{2}{|c|}{$\begin{array}{l}\text { Atomic } \\
\text { Number }\end{array}$}} & \multirow{2}{*}{$\begin{array}{r}\text { Atomic } \\
\text { Type }\end{array}$} & \multicolumn{2}{|c|}{ Coordinates (Angstrom } \\
\hline & & & & $\mathrm{X}$ & $\mathrm{Z}$ \\
\hline 1 & 40 & 0 & .000 & 04 & .000022 \\
\hline 2 & 7 & 0 & -1.045301 & -1.761263 & -1.130674 \\
\hline 3 & 7 & 0 & 1.045419 & -1.761179 & 1.130708 \\
\hline 4 & 6 & 0 & -1.376353 & -.121194 & 2.268323 \\
\hline
\end{tabular}




$\begin{array}{cccccc}5 & 6 & 0 & -.516760 & 1.026881 & 2.184724 \\ 6 & 6 & 0 & -.915585 & 1.854458 & 1.087979 \\ 7 & 6 & 0 & -2.101273 & 1.239213 & .499128 \\ 8 & 6 & 0 & -2.400373 & .024788 & 1.261171 \\ 9 & 6 & 0 & .915453 & 1.854576 & -1.087805 \\ 10 & 6 & 0 & 2.101204 & 1.239307 & -.499107 \\ 11 & 6 & 0 & 2.400273 & .024959 & -1.261285 \\ 12 & 6 & 0 & 1.376181 & -.120947 & -2.268378 \\ 13 & 6 & 0 & .516568 & 1.027095 & -2.184604 \\ 14 & 6 & 0 & -1.686534 & -2.968518 & -.474736 \\ 15 & 6 & 0 & -1.495161 & -1.782377 & -2.572576 \\ 16 & 6 & 0 & 1.687377 & -2.967983 & .474647 \\ 17 & 6 & 0 & 1.494805 & -1.782511 & 2.572753 \\ 18 & 1 & 0 & .240402 & 1.304380 & 2.927567 \\ 19 & 6 & 0 & -3.032627 & 1.689490 & -.511380 \\ 20 & 6 & 0 & -3.628703 & -.692322 & 1.005802 \\ 21 & 6 & 0 & 3.032631 & 1.689520 & .511362 \\ 22 & 1 & 0 & -1.402164 & -.836798 & 3.095439 \\ 23 & 1 & 0 & 1.348109 & -3.890447 & .987451 \\ 24 & 1 & 0 & 1.403513 & -3.028330 & -.586021 \\ 25 & 1 & 0 & 2.790083 & -2.908725 & .546057 \\ 26 & 1 & 0 & 1.079859 & -.927694 & 3.121719 \\ 27 & 1 & 0 & 1.156858 & -2.720455 & 3.058485 \\ 28 & 1 & 0 & 2.600373 & -1.737656 & 2.631747 \\ 29 & 1 & 0 & -2.600767 & -1.737961 & -2.631186 \\ 30 & 1 & 0 & -1.080753 & -.927235 & -3.121442 \\ 31 & 1 & 0 & -1.156993 & -2.720046 & -3.058685 \\ 32 & 1 & 0 & -1.402292 & -3.029041 & .585821 \\ 33 & 1 & 0 & -2.789288 & -2.909704 & -.545764 \\ 34 & 1 & 0 & -1.347049 & -3.890698 & -.987908 \\ 35 & 6 & 0 & -.309939 & 3.210774 & .723035 \\ 36 & 6 & 0 & .309819 & 3.210854 & -.722699 \\ 37 & 6 & 0 & 3.628642 & -.692143 & -1.006082 \\ 38 & 1 & 0 & -.240657 & 1.304646 & -2.927363 \\ 39 & 1 & 0 & 1.401942 & -.836476 & -3.095560 \\ 40 & 6 & 0 & -4.501086 & -.213393 & .043387 \\ 41 & 1 & 0 & .479493 & 3.444641 & 1.457454 \\ 42 & 1 & 0 & -1.079059 & 4.004729 & .789704 \\ 43 & 1 & 0 & -.479609 & 3.444818 & -1.457092 \\ 44 & 1 & 0 & 1.078946 & 4.004808 & -.789273 \\ 45 & 6 & 0 & -4.200514 & .973361 & -.718376 \\ 46 & 1 & 0 & -2.831027 & 2.613907 & -1.069230 \\ 47 & 1 & 0 & -3.874040 & -1.580049 & 1.605362 \\ 49 & 6 & 0 & 4.200556 & .973405 & .718193 \\ & 1 & 0 & 2.831053 & 2.613881 & 1.069312 \\ & 6 & 0 & 4.501094 & -.213276 & -.043697\end{array}$




$\begin{array}{lllrrl}51 & 1 & 0 & 3.873950 & -1.579812 & -1.605739 \\ 52 & 1 & 0 & -5.450435 & -.733032 & -.142605 \\ 53 & 1 & 0 & -4.928733 & 1.320212 & -1.463783 \\ 54 & 1 & 0 & 4.928829 & 1.320209 & 1.463568 \\ 55 & 1 & 0 & 5.450471 & -.732909 & .142170 \\ -\end{array}$

\section{meso-(EBI)Zr( $\left.\mathrm{NMe}_{2}\right)_{2}$ (9)}

absolute energy: -1087.40474969 hartrees imaginary frequencies: 0

\begin{tabular}{|c|c|c|c|c|c|}
\hline \multirow{2}{*}{$\begin{array}{l}\text { Center } \\
\text { Number }\end{array}$} & \multirow{2}{*}{$\begin{array}{l}\text { Atomic } \\
\text { Number }\end{array}$} & \multirow{2}{*}{\multicolumn{2}{|c|}{$\begin{array}{r}\text { Atomic } \\
\text { Type }\end{array}$}} & \multicolumn{2}{|c|}{ Coordinates (Angstrom } \\
\hline & & & & $X$ & $\mathrm{Z}$ \\
\hline 1 & 40 & 0 & -.322391 & .703628 & -.021147 \\
\hline 2 & 7 & 0 & -.270086 & .000100 & -1.958648 \\
\hline 3 & 7 & 0 & -1.295913 & 2.502757 & -.343816 \\
\hline 4 & 6 & 0 & 1.956788 & 2.068811 & -.000848 \\
\hline 5 & 6 & 0 & 1.505128 & 1.924061 & 1.355464 \\
\hline 6 & 6 & 0 & 1.623085 & .559323 & 1.767113 \\
\hline 7 & 6 & 0 & 2.292667 & -.156779 & .678835 \\
\hline 8 & 6 & 0 & 2.531807 & .797292 & -.400761 \\
\hline 9 & 6 & 0 & -.855748 & -.824108 & 2.028914 \\
\hline 10 & 6 & 0 & -1.842522 & 211056 & 2.093771 \\
\hline 11 & 6 & 0 & -2.735633 & .096329 & .978154 \\
\hline 12 & 6 & 0 & -2.405315 & -1.121323 & .266342 \\
\hline 13 & 6 & 0 & -1.217968 & -1.687296 & .897656 \\
\hline 14 & 6 & 0 & -1.251074 & 407980 & -3.034596 \\
\hline 15 & 6 & 0 & .552618 & -1.131540 & -2.525748 \\
\hline 16 & 6 & 0 & -.722977 & 3.457184 & -1.378556 \\
\hline 17 & 6 & 0 & -2.414168 & 3.259740 & .334262 \\
\hline 18 & 1 & 0 & 1.205876 & 2.751566 & 2.010976 \\
\hline 19 & 6 & 0 & 2.877500 & -1.471831 & .603199 \\
\hline 20 & 6 & 0 & 3.353407 & .418796 & -1.518486 \\
\hline 21 & 1 & 0 & -1.959257 & 916871 & 2.924888 \\
\hline 22 & 6 & 0 & -3.068160 & -1.837029 & $9-.791229$ \\
\hline 23 & 6 & 0 & -.723615 & -2.966070 & .452115 \\
\hline 24 & 1 & 0 & 2.151480 & 3.024712 & -.494730 \\
\hline 25 & 1 & 0 & -1.525502 & 3.762952 & -2.078411 \\
\hline 26 & 1 & 0 & -.316063 & 4.363134 & -.888966 \\
\hline 27 & 1 & 0 & .075888 & 2.971076 & -1.956492 \\
\hline 28 & 1 & 0 & -2.031395 & 4.223400 & .726036 \\
\hline 29 & 1 & 0 & -3.223963 & 3.474453 & $\begin{array}{ll}3 & -.391379\end{array}$ \\
\hline 30 & 1 & 0 & -2.824790 & 2.680297 & 1.169861 \\
\hline 31 & 1 & 0 & 1.260672 & -1.514003 & -1.775881 \\
\hline 32 & 1 & 0 & -.108935 & -1.960427 & -2.844409 \\
\hline
\end{tabular}




$\begin{array}{rrrccc}33 & 1 & 0 & 1.126030 & -.778271 & -3.405938 \\ 34 & 1 & 0 & -1.882227 & 1.238040 & -2.686339 \\ 35 & 1 & 0 & -.701336 & .729785 & -3.942671 \\ 36 & 1 & 0 & -1.903766 & -.446202 & -3.301644 \\ 37 & 6 & 0 & 1.299028 & .006767 & 3.155955 \\ 38 & 6 & 0 & .212782 & -1.126142 & 3.084603 \\ 39 & 1 & 0 & -3.665747 & .656941 & .847434 \\ 40 & 6 & 0 & 3.920268 & -.848395 & -1.540148 \\ 41 & 1 & 0 & .920040 & .834066 & 3.780647 \\ 42 & 1 & 0 & 2.213081 & -.392110 & 3.637840 \\ 43 & 1 & 0 & .700870 & -2.080822 & 2.827370 \\ 44 & 1 & 0 & -.257189 & -1.246466 & 4.080067 \\ 45 & 6 & 0 & 3.677628 & -1.794158 & -.485563 \\ 46 & 1 & 0 & 2.730826 & -2.187113 & 1.423538 \\ 47 & 1 & 0 & 3.554838 & 1.143783 & -2.319149 \\ 48 & 6 & 0 & -2.571752 & -3.074321 & -1.178312 \\ 49 & 1 & 0 & .150904 & -3.419176 & .938168 \\ 50 & 6 & 0 & -1.402021 & -3.634840 & -.558974 \\ 51 & 1 & 0 & -3.974563 & -1.418128 & -1.250347 \\ 52 & 1 & 0 & 4.572892 & -1.141212 & -2.373377 \\ 53 & 1 & 0 & 4.150984 & -2.783265 & -.541305 \\ 54 & 1 & 0 & -3.081337 & -3.647607 & -1.964058 \\ 55 & 1 & 0 & -1.051163 & -4.622431 & -.887589 \\ ----------------------------------------------------------------\end{array}$

\section{rac- $-\mathrm{Me}_{2} \mathrm{Si}\left(3-{ }^{\mathrm{t}} \mathrm{BuC}_{5} \mathrm{H}_{3}\right)_{2} \mathrm{Zr}\left(\mathrm{NMe}_{2}\right)_{2}(10)$ absolute energy: -1099.74471103 hartrees imaginary frequencies: 0}

\begin{tabular}{|c|c|c|c|c|c|}
\hline \multirow{2}{*}{$\begin{array}{l}\text { Center } \\
\text { Number }\end{array}$} & \multirow{2}{*}{\multicolumn{2}{|c|}{$\begin{array}{l}\text { Atomic } \\
\text { Number }\end{array}$}} & \multirow{2}{*}{$\begin{array}{r}\text { Atomic } \\
\text { Type }\end{array}$} & \multicolumn{2}{|c|}{ Coordinates (Angstrom } \\
\hline & & & & $X$ & $\mathrm{Z}$ \\
\hline 1 & 40 & 0 & -.018877 & -.362955 & .142966 \\
\hline 2 & 7 & 0 & .701746 & -1.164313 & 1.909117 \\
\hline 3 & 7 & 0 & -.371376 & -2.120386 & -.878453 \\
\hline 4 & 6 & 0 & 1.888830 & -.050598 & -1.774110 \\
\hline 5 & 6 & 0 & .941423 & 1.025706 & -1.768431 \\
\hline 6 & 6 & 0 & 1.204835 & 1.859685 & -.615385 \\
\hline 7 & 6 & 0 & 2.255053 & 1.232990 & .116183 \\
\hline 8 & 6 & 0 & 2.713104 & .053380 & -.610623 \\
\hline 9 & 6 & 0 & -1.397219 & 1.910491 & .414296 \\
\hline 10 & 6 & 0 & -2.164694 & 1.136924 & -.537301 \\
\hline 11 & 6 & 0 & -2.814119 & .051620 & .157190 \\
\hline 12 & 6 & 0 & -2.358339 & .105087 & 1.519571 \\
\hline 13 & 6 & 0 & -1.512852 & 1.258229 & 1.677898 \\
\hline 14 & 6 & 0 & -.243912 & -2.019303 & 2.736431 \\
\hline
\end{tabular}




$\begin{array}{llllll}15 & 6 & 0 & 1.903864 & -.930336 & 2.788813 \\ 16 & 6 & 0 & -.027937 & -3.529227 & -.468772 \\ 17 & 6 & 0 & -.872787 & -2.179119 & -2.299579 \\ 18 & 1 & 0 & .308581 & 1.302826 & -2.618044 \\ 19 & 6 & 0 & 4.060988 & -.714557 & -.434781 \\ 20 & 1 & 0 & -2.409016 & 1.465518 & -1.551369 \\ 21 & 1 & 0 & -2.775901 & -.477855 & 2.345052 \\ 22 & 1 & 0 & 2.041434 & -.746624 & -2.604374 \\ 23 & 14 & 0 & -.049828 & 3.214339 & -.046430 \\ 24 & 6 & 0 & -4.059077 & -.750645 & -.319738 \\ 25 & 1 & 0 & -1.126212 & 1.631665 & 2.632618 \\ 26 & 1 & 0 & 2.756425 & 1.667408 & .987328 \\ 27 & 1 & 0 & .725637 & -3.957183 & -1.160201 \\ 28 & 1 & 0 & .383322 & -3.539547 & .550133 \\ 29 & 1 & 0 & -.932276 & -4.171605 & -.499028 \\ 30 & 1 & 0 & -.091270 & -2.599031 & -2.965958 \\ 31 & 1 & 0 & -1.772280 & -2.822535 & -2.372113 \\ 32 & 1 & 0 & -1.129584 & -1.169888 & -2.655317 \\ 33 & 1 & 0 & 2.388087 & -1.895632 & 3.040062 \\ 34 & 1 & 0 & 2.625729 & -.286191 & 2.278128 \\ 35 & 1 & 0 & 1.591603 & -.440735 & 3.733479 \\ 36 & 1 & 0 & -1.137841 & -2.291230 & 2.157758 \\ 37 & 1 & 0 & .276940 & -2.952461 & 3.028318 \\ 38 & 1 & 0 & -.552805 & -1.483808 & 3.654858 \\ 39 & 6 & 0 & -.590288 & 4.407226 & -1.529206 \\ 40 & 6 & 0 & .582232 & 4.219445 & 1.527037 \\ 41 & 6 & 0 & 4.948302 & -.070786 & .685495 \\ 42 & 6 & 0 & 3.827438 & -2.232532 & -.121521 \\ 43 & 6 & 0 & 4.856996 & -.601599 & -1.790758 \\ 44 & 6 & 0 & -5.314548 & -.106054 & .383072 \\ 45 & 6 & 0 & -4.259386 & -.648658 & -1.868113 \\ 46 & 6 & 0 & -3.968760 & -2.258059 & .098123 \\ 47 & 1 & 0 & 5.919356 & -.587142 & .726254 \\ 48 & 1 & 0 & 5.130501 & .993433 & .473143 \\ 49 & 1 & 0 & 4.476418 & -.154385 & 1.674191 \\ 50 & 1 & 0 & 3.327180 & -2.354292 & .849722 \\ 51 & 1 & 0 & 3.197277 & -2.688162 & -.898198 \\ 52 & 1 & 0 & 4.791577 & -2.765463 & -.091318 \\ 53 & 1 & 0 & 5.842977 & -1.079799 & -1.684889 \\ 54 & 1 & 0 & 4.315250 & -1.103262 & -2.605653 \\ 55 & 1 & 0 & 5.007615 & .453416 & -2.064689 \\ 56 & 1 & 0 & -4.883109 & -2.789580 & -.208104 \\ 57 & 1 & 0 & -3.102752 & -2.736027 & -.383062 \\ 50 & 1 & 0 & -3.860402 & -2.353167 & 1.188089 \\ 60 & 1 & 0 & -5.228917 & -.182361 & 1.476784 \\ & 1 & 0 & -5.403372 & .957573 & .113513\end{array}$




$\begin{array}{llllll}61 & 1 & 0 & -6.232587 & -.623978 & .065776 \\ 62 & 1 & 0 & -3.399772 & -1.071970 & -2.405065 \\ 63 & 1 & 0 & -5.162754 & -1.205006 & -2.161970 \\ 64 & 1 & 0 & -4.385601 & .399486 & -2.176300 \\ 65 & 1 & 0 & 1.446294 & 4.842763 & 1.268694 \\ 66 & 1 & 0 & -.211391 & 4.869576 & 1.912712 \\ 67 & 1 & 0 & .879714 & 3.525778 & 2.322139 \\ 68 & 1 & 0 & .251407 & 5.031186 & -1.849591 \\ 69 & 1 & 0 & -.931797 & 3.818107 & -2.388166 \\ 70 & 1 & 0 & -1.408474 & 5.064664 & -1.213368\end{array}$

\section{meso- $\mathrm{Me}_{2} \mathrm{Si}\left(3-{ }^{\mathrm{t}} \mathrm{BuC}_{5} \mathrm{H}_{3}\right)_{2} \mathrm{Zr}\left(\mathrm{NMe}_{2}\right)_{2}$ (10) absolute energy: $-\mathbf{1 0 9 9 . 7 4 6 8 4 1 8 5}$ hartrees imaginary frequencies: 0}

\begin{tabular}{|c|c|c|c|c|c|}
\hline \multirow{2}{*}{$\begin{array}{l}\text { Center } \\
\text { Number }\end{array}$} & \multirow{2}{*}{$\begin{array}{l}\text { Atomic } \\
\text { Number }\end{array}$} & \multirow{2}{*}{\multicolumn{2}{|c|}{$\begin{array}{l}\text { Atomic } \\
\text { Type }\end{array}$}} & \multicolumn{2}{|c|}{ Coordinates (Angstrom } \\
\hline & & & & $\mathrm{X}$ & $\mathrm{Z}$ \\
\hline 1 & 40 & 0 & .068623 & -.287567 & .452783 \\
\hline 2 & 7 & 0 & -.070212 & -1.875990 & -.873722 \\
\hline 3 & 7 & 0 & .189566 & -1.426464 & 2.178951 \\
\hline 4 & 6 & 0 & -2.525668 & .245533 & 1.240742 \\
\hline 5 & 6 & 0 & -1.692908 & 1.404511 & 1.431754 \\
\hline 6 & 6 & 0 & -1.369469 & 1.942023 & .144606 \\
\hline 7 & 6 & 0 & -1.958584 & 1.071960 & -.839788 \\
\hline 8 & 6 & 0 & -2.738948 & .058 & -.163765 \\
\hline 9 & 6 & 0 & 1.416730 & 1.966407 & .118628 \\
\hline 10 & 6 & ( & 1.878760 & 1.482981 & 1.377912 \\
\hline 11 & 6 & 0 & 2.657540 & .294424 & 1.144474 \\
\hline 12 & 6 & 0 & 2.703105 & .030087 & -.264997 \\
\hline 13 & 6 & 0 & 1.876656 & 1.035999 & -.890650 \\
\hline 14 & 6 & 0 & .361879 & -3.298383 & -.608503 \\
\hline 15 & 6 & 0 & -.458465 & -1.814858 & -2.330709 \\
\hline 16 & 6 & 0 & -.949048 & -2.388892 & 2.473943 \\
\hline 17 & 6 & 0 & 1.056144 & -1.444564 & 3.417271 \\
\hline 18 & 1 & 0 & -1.503058 & 1.887085 & 2.396493 \\
\hline 19 & 6 & 0 & -3.894443 & -.794829 & -.768220 \\
\hline 20 & 1 & 0 & 1.788112 & 1.998093 & 2.339793 \\
\hline 21 & 6 & 0 & 3.764122 & -.869079 & -.958889 \\
\hline 22 & 1 & 0 & -3.077186 & -.259930 & 2.039346 \\
\hline 23 & 1 & 0 & -.532412 & -3.391555 & 2.693188 \\
\hline 24 & 1 & 0 & -1.528492 & -2.046553 & 3.352930 \\
\hline 25 & 1 & 0 & -1.621772 & -2.473081 & 1.609588 \\
\hline 26 & 1 & 0 & 1.859933 & -.704934 & 3.338356 \\
\hline 27 & 1 & 0 & .441108 & -1.207461 & 4.308444 \\
\hline
\end{tabular}




\begin{tabular}{|c|c|c|c|c|c|}
\hline 28 & 1 & 0 & 1.499394 & -2.451058 & 3.555069 \\
\hline 29 & 1 & 0 & -.766442 & -.795747 & -2.603572 \\
\hline 30 & 1 & 0 & .395315 & -2.105962 & -2.974044 \\
\hline 31 & 1 & 0 & -1.294962 & -2.513067 & -2.536717 \\
\hline 32 & 1 & 0 & .700071 & -3.407438 & .430986 \\
\hline 33 & 1 & 0 & -.487325 & -3.990248 & -.785775 \\
\hline 34 & 1 & 0 & 1.189484 & -3.589846 & -1.285362 \\
\hline 35 & 14 & 0 & .007493 & 3.257608 & -.147800 \\
\hline 36 & 1 & 0 & 3.292947 & -.196359 & 1.887490 \\
\hline 37 & 1 & 0 & 1.804482 & 1.203389 & -1.970115 \\
\hline 38 & 1 & 0 & -2.011204 & 1.293613 & -1.909647 \\
\hline 39 & 6 & 0 & -3.784557 & -2.304878 & -.365797 \\
\hline 40 & 6 & 0 & 4.012944 & -2.187373 & -.151533 \\
\hline 41 & 6 & 0 & 3.360559 & -1.228317 & -2.426535 \\
\hline 42 & 6 & 0 & 5.103057 & -.036577 & -.996547 \\
\hline 43 & 6 & 0 & -5.244140 & -.220330 & -.189308 \\
\hline 44 & 6 & 0 & -3.952756 & -.671625 & -2.328427 \\
\hline 45 & 6 & 0 & -.007799 & 3.990237 & -1.980782 \\
\hline 46 & 6 & 0 & .014202 & 4.684413 & 1.220354 \\
\hline 47 & 1 & 0 & 3.201638 & -.319433 & -3.024721 \\
\hline 48 & 1 & 0 & 4.161718 & -1.817569 & -2.898716 \\
\hline 49 & 1 & 0 & 2.436276 & -1.822319 & -2.436575 \\
\hline 50 & 1 & 0 & 4.362498 & -1.963949 & .866650 \\
\hline 51 & 1 & 0 & 3.086274 & -2.775838 & -.082752 \\
\hline 52 & 1 & 0 & 4.780465 & -2.795442 & -.654870 \\
\hline 53 & 1 & 0 & 4.961502 & .895668 & -1.564437 \\
\hline 54 & 1 & 0 & 5.425875 & .221320 & .023561 \\
\hline 55 & 1 & 0 & 5.900279 & -.622877 & -1.478791 \\
\hline 56 & 1 & 0 & -2.851960 & -2.740088 & -.753824 \\
\hline 57 & 1 & 0 & -3.795853 & -2.413901 & .728632 \\
\hline 58 & 1 & 0 & -4.636040 & -2.868359 & -.778789 \\
\hline 59 & 1 & 0 & -5.272787 & -.322051 & .905611 \\
\hline 60 & 1 & 0 & -5.350174 & .845274 & -.443865 \\
\hline 61 & 1 & 0 & -6.099758 & -.768183 & -.613466 \\
\hline 62 & 1 & 0 & -3.035350 & -1.056677 & -2.792755 \\
\hline 63 & 1 & 0 & -4.805618 & -1.252223 & -2.711542 \\
\hline 64 & 1 & 0 & -4.086957 & .377393 & -2.630508 \\
\hline 65 & 1 & 0 & -.874598 & 5.317444 & 1.117924 \\
\hline 66 & 1 & 0 & .016495 & 4.251219 & 2.227064 \\
\hline 67 & 1 & 0 & .903633 & 5.315637 & 1.112420 \\
\hline 68 & 1 & 0 & -.021732 & 3.177789 & -2.716270 \\
\hline 69 & 1 & 0 & -.895106 & 4.614158 & -2.137716 \\
\hline 70 & 1 & 0 & .882498 & 4.604090 & -2.158840 \\
\hline
\end{tabular}




\section{rac-Me $\mathrm{Me}_{2} \mathrm{Si}\left(3^{\mathrm{t}}{ }^{\mathrm{t}} \mathrm{Bu}-\mathrm{C}_{5} \mathrm{H}_{3}\right) \mathrm{Zr}\left\{\mathrm{PhN}\left(\mathrm{CH}_{2}\right)_{3} \mathrm{NPh}\right\}$ (11)

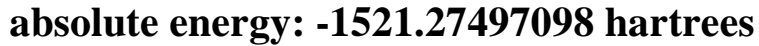 imaginary frequencies: 0}

\begin{tabular}{|c|c|c|c|c|c|}
\hline \multirow{2}{*}{$\begin{array}{l}\text { Center } \\
\text { Number }\end{array}$} & \multirow{2}{*}{\multicolumn{2}{|c|}{$\begin{array}{l}\text { Atomic } \\
\text { Number }\end{array}$}} & \multirow{2}{*}{$\begin{array}{l}\text { Atomic } \\
\text { Type }\end{array}$} & \multicolumn{2}{|c|}{ Coordinates (Angstroms } \\
\hline & & & & $X$ & $\mathrm{Z}$ \\
\hline 1 & 40 & 0 & .040855 & .026155 & .162621 \\
\hline 2 & 7 & 0 & -1.455726 & .670699 & -1.141364 \\
\hline 3 & 7 & 0 & 1.055901 & -.721946 & -1.504962 \\
\hline 4 & 6 & 0 & -.242378 & -1.085482 & 2.532594 \\
\hline 5 & 6 & 0 & .126674 & -2.149897 & 1.634121 \\
\hline 6 & 6 & 0 & -.996357 & -2.461011 & .775046 \\
\hline 7 & 6 & 0 & -2.026433 & -1.516385 & 1.103785 \\
\hline 8 & 6 & 0 & -1.564946 & -.671785 & 2.171165 \\
\hline 9 & 6 & 0 & 1.407478 & 1.117209 & 2.153548 \\
\hline 10 & 6 & 0 & 2.364672 & .883232 & 1.115379 \\
\hline 11 & 6 & 0 & 2.112186 & 1.817836 & .047376 \\
\hline 12 & 6 & 0 & .978375 & 2.620025 & .389557 \\
\hline 13 & 6 & 0 & .507551 & 2.129915 & 1.675159 \\
\hline 14 & 6 & 0 & .196334 & -1.186321 & -2.676550 \\
\hline 15 & 6 & 0 & -.966360 & 1.145677 & -2.506943 \\
\hline 16 & 6 & 0 & -2.918954 & .740346 & -1.048880 \\
\hline 17 & 6 & 0 & 2.484004 & -.864589 & -1.817283 \\
\hline 18 & 1 & 0 & -3.053972 & -1.539142 & .727379 \\
\hline 19 & 1 & 0 & 2.778406 & 1.982983 & -.805264 \\
\hline 20 & 14 & 0 & .992304 & -.073111 & 3.614953 \\
\hline 21 & 1 & 0 & 3.252239 & .246978 & 1.190649 \\
\hline 22 & 1 & 0 & 1.022575 & -2.769990 & 1.738224 \\
\hline 23 & 6 & 0 & .614116 & 4.014274 & -.199731 \\
\hline 24 & 1 & 0 & -2.185522 & .044288 & 2.718790 \\
\hline 25 & 1 & 0 & -.258384 & 2.622101 & 2.283750 \\
\hline 26 & 6 & 0 & -1.204704 & -3.818323 & .043973 \\
\hline 27 & 1 & 0 & -.589302 & -1.851451 & -2.284498 \\
\hline 28 & 1 & 0 & .820471 & -1.775657 & -3.376881 \\
\hline 29 & 6 & 0 & -.499672 & -.014392 & -3.451118 \\
\hline 30 & 6 & 0 & -3.534255 & 1.361273 & .071450 \\
\hline 31 & 6 & 0 & -3.756034 & .209017 & -2.071308 \\
\hline 32 & 6 & 0 & 3.340033 & -1.590384 & -.948236 \\
\hline 33 & 6 & 0 & 3.041856 & -.296732 & -2.999490 \\
\hline 34 & 1 & 0 & -1.767051 & 1.732212 & -3.000983 \\
\hline 35 & 1 & 0 & -.109390 & 1.818536 & -2.343257 \\
\hline 36 & 6 & 0 & 2.551567 & -1.138540 & 4.186024 \\
\hline 37 & 6 & 0 & .113016 & .841377 & 5.128946 \\
\hline 38 & 6 & 0 & 1.272231 & 4.241085 & -1.603166 \\
\hline 39 & 6 & 0 & -.933236 & 4.226145 & -.303707 \\
\hline
\end{tabular}




$\begin{array}{lllccc}40 & 6 & 0 & 1.202746 & 5.087667 & .794805 \\ 41 & 6 & 0 & -1.637218 & -4.856476 & 1.150757 \\ 42 & 6 & 0 & -2.349002 & -3.736899 & -1.022395 \\ 43 & 6 & 0 & .113309 & -4.332477 & -.626521 \\ 44 & 1 & 0 & -1.360960 & -.434731 & -3.997185 \\ 45 & 1 & 0 & .191679 & .412861 & -4.196644 \\ 46 & 1 & 0 & -2.895803 & 1.811144 & .843049 \\ 47 & 6 & 0 & -4.935864 & 1.424688 & .178161 \\ 48 & 1 & 0 & -3.305605 & -.280663 & -2.943941 \\ 49 & 6 & 0 & -5.154839 & .283982 & -1.960505 \\ 50 & 1 & 0 & 2.908748 & -2.066567 & -.058034 \\ 51 & 6 & 0 & 4.711903 & -1.722640 & -1.235698 \\ 52 & 1 & 0 & 2.399874 & .272906 & -3.682431 \\ 53 & 6 & 0 & 4.410437 & -.440365 & -3.282397 \\ 54 & 1 & 0 & 2.243761 & -1.937759 & 4.870865 \\ 55 & 1 & 0 & 3.039065 & -1.594087 & 3.316527 \\ 56 & 1 & 0 & 3.281689 & -.504414 & 4.700216 \\ 57 & 1 & 0 & -.242732 & .110566 & 5.864212 \\ 58 & 1 & 0 & .813032 & 1.521645 & 5.626876 \\ 59 & 1 & 0 & -.746902 & 1.424644 & 4.778088 \\ 60 & 1 & 0 & .744112 & 4.984902 & 1.790539 \\ 61 & 1 & 0 & 2.291381 & 4.963175 & .897068 \\ 62 & 1 & 0 & .999098 & 6.102109 & .419226 \\ 63 & 1 & 0 & -1.149676 & 5.245354 & -.659047 \\ 64 & 1 & 0 & -1.377075 & 3.505705 & -1.005073 \\ 65 & 1 & 0 & -1.409061 & 4.092177 & .678729 \\ 66 & 1 & 0 & .919421 & 3.499101 & -2.333230 \\ 67 & 1 & 0 & 1.010024 & 5.242851 & -1.975419 \\ 68 & 1 & 0 & 2.367718 & 4.173633 & -1.536590 \\ 69 & 1 & 0 & .461837 & -3.629355 & -1.395996 \\ 70 & 1 & 0 & .908171 & -4.449746 & .123767 \\ 71 & 1 & 0 & -.065132 & -5.311157 & -1.097531 \\ 72 & 1 & 0 & -1.806710 & -5.844737 & .696751 \\ 73 & 1 & 0 & -.853671 & -4.952058 & 1.916750 \\ 74 & 1 & 0 & -2.568269 & -4.531958 & 1.641908 \\ 75 & 1 & 0 & -2.111845 & -2.998007 & -1.801615 \\ 76 & 1 & 0 & -2.480781 & -4.718867 & -1.502171 \\ 77 & 1 & 0 & -3.301238 & -3.450456 & -.552959 \\ 78 & 1 & 0 & -5.391108 & 1.911346 & 1.051625 \\ 79 & 6 & 0 & -5.752671 & .886970 & -.835483 \\ 80 & 1 & 0 & -5.782984 & -.138976 & -2.756239 \\ 81 & 1 & 0 & 5.356978 & -2.291453 & -.551096 \\ 85 & 6 & 0 & 5.253422 & -1.149719 & -2.401456 \\ & 1 & 0 & 4.824391 & .010551 & -4.194006 \\ 84 & 1 & 0 & -6.845709 & .942627 & -.753854 \\ 53 & 0 & 6.322620 & -1.256895 & -2.626833\end{array}$




\section{meso-Me $2 \mathrm{Si}\left(3^{\mathrm{t}}{ }^{\mathrm{B}} \mathrm{Bu}-\mathrm{C}_{5} \mathrm{H}_{3}\right) \mathrm{Zr}\left\{\mathrm{PhN}\left(\mathrm{CH}_{2}\right)_{3} \mathrm{NPh}\right\}$ (11) absolute energy: -1521.27440274 hartrees imaginary frequencies: 0}

\begin{tabular}{|c|c|c|c|c|c|}
\hline \multirow{2}{*}{$\begin{array}{l}\text { Center } \\
\text { Number }\end{array}$} & \multirow{2}{*}{$\begin{array}{l}\text { Atomic } \\
\text { Number }\end{array}$} & \multirow{2}{*}{\multicolumn{2}{|c|}{$\begin{array}{l}\text { Atomic } \\
\text { Type }\end{array}$}} & \multicolumn{2}{|c|}{ Coordinates (Angstroms } \\
\hline & & & & $X$ & $\mathrm{Z}$ \\
\hline 1 & 6 & 0 & -2.907290 & -1.009706 & -1.632856 \\
\hline 2 & 6 & 0 & -3.579029 & .134887 & -2.137250 \\
\hline 3 & 6 & 0 & -3.657670 & -2.201593 & -1.422745 \\
\hline 4 & 7 & 0 & -1.465457 & -.958363 & -1.360567 \\
\hline 5 & 1 & 0 & -2.996321 & 1.043692 & -2.338071 \\
\hline 6 & 6 & 0 & -4.961753 & .097927 & -2.397038 \\
\hline 7 & 6 & 0 & -5.036332 & -2.231396 & -1.692323 \\
\hline 8 & 1 & 0 & -3.157618 & -3.095858 & -1.030125 \\
\hline 9 & 6 & 0 & -.730220 & -2.096037 & -2.064146 \\
\hline 10 & 40 & 0 & -.219713 & .252666 & -.221638 \\
\hline 11 & 1 & 0 & -5.463730 & .993376 & -2.787806 \\
\hline 12 & 6 & 0 & -5.6963 & -1.082963 & $3-2.175516$ \\
\hline 13 & 1 & 0 & -5.601945 & -3.156691 & -1.517218 \\
\hline 14 & 1 & 0 & .171551 & -1.674550 & -2.538065 \\
\hline 15 & 1 & 0 & -1.375624 & -2.502034 & $4 \quad-2.867690$ \\
\hline 16 & 6 & 0 & -.288134 & -3.248877 & -1.091847 \\
\hline 17 & 7 & 0 & .805504 & -1.464657 & .427935 \\
\hline 18 & 1 & 0 & -6.773851 & -1.111445 & -2.382698 \\
\hline 19 & 1 & 0 & -1.070025 & -4.026257 & $7 \quad-1.044247$ \\
\hline 20 & 1 & 0 & .624677 & -3.713755 & -1.501789 \\
\hline 21 & 6 & 0 & -.027413 & -2.742958 & .363885 \\
\hline 22 & 6 & 0 & 2.131065 & -1.706975 & .986657 \\
\hline 23 & 6 & 0 & 2.109976 & 1.638303 & -.690902 \\
\hline 24 & 6 & 0 & 2.125649 & .723399 & -1.816482 \\
\hline 25 & 6 & 0 & .942185 & .992262 & -2.580000 \\
\hline 26 & 6 & 0 & .244102 & 2.094226 & -1.972359 \\
\hline 27 & 6 & 0 & .991471 & 2.526604 & -.823406 \\
\hline 28 & 6 & 0 & -.937665 & 2.356649 & 1.175652 \\
\hline 29 & 6 & 0 & -2.137722 & 1.932398 & .521473 \\
\hline 30 & 6 & 0 & -2.559938 & .689468 & 1.106967 \\
\hline 31 & 6 & 0 & -1.628676 & .317392 & 2.136068 \\
\hline 32 & 6 & 0 & -.598318 & 1.333259 & 2.133662 \\
\hline 33 & 6 & 0 & 2.869894 & -2.897790 & .709878 \\
\hline 34 & 6 & 0 & 2.720912 & -.747646 & 1.857675 \\
\hline 35 & 1 & 0 & 2.936306 & 1.772583 & .012554 \\
\hline 36 & 1 & 0 & .714915 & .584558 & -3.570506 \\
\hline 37 & 1 & 0 & -.591782 & 2.633201 & -2.430838 \\
\hline 38 & 6 & 0 & 3.362328 & -.055818 & -2.357115 \\
\hline
\end{tabular}




\begin{tabular}{lccccc}
39 & 1 & 0 & -2.725195 & 2.524301 & -.186891 \\
40 & 1 & 0 & -3.523701 & .206903 & .917409 \\
41 & 1 & 0 & .187378 & 1.420995 & 2.890156 \\
42 & 6 & 0 & -1.901888 & -.669918 & 3.306782 \\
43 & 14 & 0 & .265830 & 3.713820 & .511454 \\
44 & 1 & 0 & -1.003070 & -2.545588 & .838445 \\
45 & 1 & 0 & .471458 & -3.537386 & .951359 \\
46 & 1 & 0 & 2.456287 & -3.656193 & .034992 \\
47 & 6 & 0 & 4.137489 & -3.103543 & 1.279184 \\
48 & 6 & 0 & 3.994132 & -.959034 & 2.414726 \\
49 & 1 & 0 & 2.147692 & .151462 & 2.117210 \\
50 & 6 & 0 & 3.861588 & .718253 & -3.637246 \\
51 & 6 & 0 & 3.003826 & -1.522837 & -2.768366 \\
52 & 6 & 0 & 4.528620 & -.077909 & -1.313976 \\
53 & 6 & 0 & -.586067 & -1.338479 & 3.829186 \\
54 & 6 & 0 & -2.931692 & -1.778211 & 2.903217 \\
55 & 6 & 0 & -2.528096 & .186227 & 4.474366 \\
56 & 6 & 0 & 1.600631 & 4.274954 & 1.850573 \\
57 & 6 & 0 & -.689485 & 5.253246 & -.278175 \\
58 & 1 & 0 & 4.687371 & -4.025989 & 1.047631 \\
59 & 6 & 0 & 4.710268 & -2.138834 & 2.131965 \\
60 & 1 & 0 & 4.420822 & -.204449 & 3.089256 \\
61 & 1 & 0 & 3.087797 & .718910 & -4.419433 \\
62 & 1 & 0 & 4.765224 & .235333 & -4.040066 \\
63 & 1 & 0 & 4.105900 & 1.761885 & -3.386335 \\
64 & 1 & 0 & 2.672965 & -2.093828 & -1.888930 \\
65 & 1 & 0 & 3.888988 & -2.018852 & -3.196127 \\
66 & 1 & 0 & 2.204406 & -1.530251 & -3.523972 \\
67 & 1 & 0 & 4.854073 & .944805 & -1.073695 \\
68 & 1 & 0 & 5.387263 & -.622796 & -1.734316 \\
69 & 1 & 0 & 4.216637 & -.577191 & -.386893 \\
70 & 1 & 0 & -.123250 & -1.951549 & 3.043232 \\
71 & 1 & 0 & -.812557 & -1.982912 & 4.692683 \\
72 & 1 & 0 & .138930 & -.575609 & 4.147017 \\
73 & 1 & 0 & -3.895185 & -1.332678 & 2.616120 \\
74 & 1 & 0 & -3.103411 & -2.452630 & 3.755963 \\
75 & 1 & 0 & -2.557971 & -2.375078 & 2.058100 \\
76 & 1 & 0 & -1.822760 & .964431 & 4.803378 \\
77 & 1 & 0 & -2.761768 & -.460592 & 5.334004 \\
78 & 1 & 0 & -3.456456 & .674326 & 4.140173 \\
79 & 1 & 0 & 2.104845 & 3.401507 & 2.279692 \\
80 & 1 & 0 & 1.120525 & 4.832921 & 2.662574 \\
81 & 1 & 0 & 2.357648 & 4.919018 & 1.388645 \\
83 & 1 & 0 & .016668 & 5.925904 & -.778262 \\
\hline & 1 & 0 & -1.217172 & 5.818711 & .498355 \\
53 & 0 & -1.422905 & 4.910158 & -1.016638
\end{tabular}


$\begin{array}{llllll}85 & 1 & 0 & 5.700188 & -2.307370 & 2.574240\end{array}$

\section{rac-(EBI)Zr(pyrrolidide) 2 absolute energy: $\mathbf{- 1 2 4 2 . 2 2 2 8 5 4 5 3}$ imaginary frequencies: 0}

\begin{tabular}{cccccc} 
Center & \multicolumn{2}{c}{ Atomic } & Atomic & \multicolumn{3}{c}{ Coordinates } & (Angstroms) \\
Number & Number & Type & X & Y & $Z$ \\
--------------- & Z \\
1 & 40 & 0 & .072898 & .007015 & .031972 \\
2 & 7 & 0 & -1.096371 & 1.279681 & 1.167645 \\
3 & 7 & 0 & 1.280087 & 1.488896 & -.751774 \\
4 & 6 & 0 & -.949685 & .034707 & -2.417604 \\
5 & 6 & 0 & -.052935 & -1.087081 & -2.365905 \\
6 & 6 & 0 & -.567967 & -2.080116 & -1.475612 \\
7 & 6 & 0 & -1.857255 & -1.597227 & -.995157 \\
8 & 6 & 0 & -2.102638 & -.291425 & -1.611265 \\
9 & 6 & 0 & .888240 & -2.319679 & .953138 \\
10 & 6 & 0 & 2.134155 & -1.605921 & .678540 \\
11 & 6 & 0 & 2.246711 & -.514436 & 1.647155 \\
12 & 6 & 0 & 1.059441 & -.535759 & 2.468088 \\
13 & 6 & 0 & .275037 & -1.671166 & 2.071541 \\
14 & 6 & 0 & -1.739723 & 2.546140 & .608344 \\
15 & 6 & 0 & -1.704547 & 1.203512 & 2.557038 \\
16 & 6 & 0 & 1.796335 & 2.671284 & .057161 \\
17 & 6 & 0 & 1.954314 & 1.722762 & -2.091458 \\
18 & 1 & 0 & .822590 & -1.226515 & -3.010812 \\
19 & 6 & 0 & -2.909718 & -2.227037 & -.227598 \\
20 & 6 & 0 & -3.400503 & .329816 & -1.471314 \\
21 & 6 & 0 & 3.245857 & -1.889879 & -.202220 \\
22 & 1 & 0 & -.894436 & .859438 & -3.133798 \\
23 & 6 & 0 & .052974 & -3.451354 & -1.202544 \\
24 & 6 & 0 & .425802 & -3.631010 & .314964 \\
25 & 6 & 0 & 3.468529 & .251100 & 1.719467 \\
26 & 1 & 0 & .911488 & .053455 & 3.376263 \\
27 & 1 & 0 & -.587668 & -2.065118 & 2.622665 \\
28 & 1 & 0 & 2.850889 & 2.488080 & .346695 \\
29 & 6 & 0 & 1.716296 & 3.898721 & -.920015 \\
30 & 1 & 0 & 1.197530 & 2.811030 & .972058 \\
31 & 6 & 0 & 1.823221 & 3.264944 & -2.352209 \\
32 & 1 & 0 & 3.025220 & 1.443989 & -2.018510 \\
33 & 1 & 0 & 1.485692 & 1.116452 & -2.881450
\end{tabular}




$\begin{array}{cccccc}34 & 1 & 0 & -1.572240 & .202310 & 2.992165 \\ 35 & 1 & 0 & -1.209208 & 1.951472 & 3.212157 \\ 36 & 1 & 0 & -1.718701 & 2.543307 & -.494002 \\ 37 & 6 & 0 & -3.202617 & 2.609133 & 1.185762 \\ 38 & 1 & 0 & -1.159716 & 3.422327 & .964755 \\ 39 & 6 & 0 & -3.210415 & 1.578813 & 2.365115 \\ 40 & 6 & 0 & -4.385672 & -.321791 & -.750256 \\ 41 & 1 & 0 & .963681 & -3.548218 & -1.817051 \\ 42 & 1 & 0 & -.645269 & -4.256015 & -1.504089 \\ 43 & 1 & 0 & -.458867 & -3.990506 & .866777 \\ 44 & 1 & 0 & 1.216445 & -4.401405 & .406278 \\ 45 & 6 & 0 & -4.137679 & -1.595883 & -.121473 \\ 46 & 1 & 0 & -2.744134 & -3.216963 & .218229 \\ 47 & 1 & 0 & -3.602135 & 1.285191 & -1.975628 \\ 48 & 6 & 0 & 4.401323 & -1.131726 & -.093439 \\ 49 & 1 & 0 & 3.184205 & -2.725507 & -.911669 \\ 50 & 6 & 0 & 4.514949 & -.065102 & .869541 \\ 51 & 1 & 0 & 3.569171 & 1.044646 & 2.474337 \\ 52 & 1 & 0 & -3.434774 & 3.628137 & 1.534713 \\ 53 & 1 & 0 & -3.938655 & 2.330490 & .416077 \\ 54 & 1 & 0 & -3.782562 & .679268 & 2.084854 \\ 55 & 1 & 0 & -3.646382 & 1.998534 & 3.286877 \\ 56 & 1 & 0 & .914669 & 3.476030 & -2.939663 \\ 57 & 1 & 0 & 2.692149 & 3.648825 & -2.911136 \\ 58 & 1 & 0 & .756396 & 4.423915 & -.796094 \\ 59 & 1 & 0 & 2.529706 & 4.614546 & -.722650 \\ 60 & 1 & 0 & -5.385531 & .124080 & -.660528 \\ 61 & 1 & 0 & -4.954337 & -2.078757 & .432856 \\ 62 & 1 & 0 & 5.262333 & -1.354630 & -.738051 \\ 63 & 1 & 0 & 5.459545 & .491442 & .930694 \\ ------------------------------------------------------------- \\ & & & & & \\ 54 & & & & \\ 53 & & 0 & \end{array}$

\section{meso-(EBI)Zr(pyrrolidide)2}

absolute energy: -1242.22045178

imaginary frequencies: 1 (-24.2454)

\begin{tabular}{cccccc} 
Center & \multicolumn{2}{c}{ Atomic } & \multicolumn{2}{c}{ Atomic } & \multicolumn{2}{c}{ Coordinates (Angstroms) } \\
Number & Number & Type & X & Y & Z \\
\hline 1 & 40 & 0 & -.563296 & -.131771 & -.109881 \\
2 & 7 & 0 & .388960 & .305910 & 1.655227 \\
3 & 7 & 0 & -2.489866 & -.089771 & .642277 \\
4 & 6 & 0 & -.988040 & -2.736568 & .038492 \\
5 & 6 & 0 & -1.160433 & -2.391417 & -1.348753 \\
6 & 6 & 0 & .079887 & -1.955412 & -1.897583 \\
7 & 6 & 0 & 1.093986 & -2.124464 & -.850578
\end{tabular}




$\begin{array}{cccccc}8 & 6 & 0 & .425982 & -2.655741 & .335527 \\ 9 & 6 & 0 & .387755 & .840047 & -2.430780 \\ 10 & 6 & 0 & -.968550 & 1.261655 & -2.273979 \\ 11 & 6 & 0 & -1.061788 & 2.226001 & -1.209550 \\ 12 & 6 & 0 & .292341 & 2.545183 & -.797107 \\ 13 & 6 & 0 & 1.195668 & 1.656107 & -1.522525 \\ 14 & 6 & 0 & -.182874 & .027947 & 3.048219 \\ 15 & 6 & 0 & 1.858280 & .698205 & 1.864506 \\ 16 & 6 & 0 & -3.117567 & 1.179610 & 1.214490 \\ 17 & 6 & 0 & -3.700187 & -1.009788 & .608212 \\ 18 & 1 & 0 & -2.082200 & -2.533864 & -1.925475 \\ 19 & 6 & 0 & 2.534621 & -2.028831 & -.864251 \\ 20 & 6 & 0 & 1.205630 & -3.079341 & 1.470283 \\ 21 & 1 & 0 & -1.798582 & .975863 & -2.931987 \\ 22 & 6 & 0 & .833699 & 3.609037 & .006993 \\ 23 & 6 & 0 & 2.619477 & 1.865247 & -1.430664 \\ 24 & 1 & 0 & -1.724544 & -3.254697 & .659259 \\ 25 & 1 & 0 & -2.447092 & 2.044885 & 1.098446 \\ 26 & 6 & 0 & -4.483433 & 1.342630 & .465874 \\ 27 & 1 & 0 & -3.302447 & 1.018847 & 2.296858 \\ 28 & 6 & 0 & -4.882496 & -.124565 & .075674 \\ 29 & 1 & 0 & -3.515261 & -1.888764 & -.024465 \\ 30 & 1 & 0 & -3.919555 & -1.350524 & 1.641821 \\ 31 & 1 & 0 & 2.523762 & .065794 & 1.250401 \\ 32 & 1 & 0 & 1.992741 & 1.749156 & 1.554131 \\ 33 & 1 & 0 & -.181280 & -1.061549 & 3.248203 \\ 34 & 6 & 0 & .772240 & .744073 & 4.052411 \\ 35 & 1 & 0 & -1.220744 & .392204 & 3.116594 \\ 36 & 6 & 0 & .323961 & -1.564429 & -3.356588 \\ 37 & 6 & 0 & .926816 & -.118764 & -3.495555 \\ 38 & 6 & 0 & 2.162492 & .539727 & 3.389689 \\ 39 & 1 & 0 & -1.934912 & 2.857560 & -1.024795 \\ 40 & 6 & 0 & 2.587984 & -2.985470 & 1.409173 \\ 41 & 1 & 0 & -.642224 & -1.606792 & -3.887928 \\ 42 & 1 & 0 & 1.006497 & -2.290880 & -3.839771 \\ 43 & 1 & 0 & 2.023704 & -.170674 & -3.398298 \\ 44 & 1 & 0 & .701018 & .268193 & -4.508625 \\ 45 & 6 & 0 & 3.249889 & -2.457373 & .245546 \\ 46 & 1 & 0 & 3.053076 & -1.666701 & -1.761895 \\ 47 & 1 & 0 & .702090 & -3.505258 & 2.349370 \\ 48 & 6 & 0 & 2.209133 & 3.796783 & .041492 \\ 49 & 1 & 0 & 3.307315 & 1.222093 & -1.996061 \\ 50 & 6 & 0 & 3.100755 & 2.925572 & -.675878 \\ 51 & 1 & 0 & .156416 & 4.289679 & .541286 \\ 52 & 1 & 0 & .527188 & 1.817811 & 4.114193 \\ 53 & 1 & 0 & .709451 & .306206 & 5.061746\end{array}$




$\begin{array}{lllllc}54 & 1 & 0 & 2.537727 & -.475434 & 3.601678 \\ 55 & 1 & 0 & 2.912597 & 1.270957 & 3.731655 \\ 56 & 1 & 0 & -5.840286 & -.428399 & .528134 \\ 57 & 1 & 0 & -4.968829 & -.227636 & -1.017901 \\ 58 & 1 & 0 & -5.237693 & 1.823020 & 1.110027 \\ 59 & 1 & 0 & -4.350937 & 1.963762 & -.434480 \\ 60 & 1 & 0 & 3.198895 & -3.329798 & 2.254168 \\ 61 & 1 & 0 & 4.347292 & -2.414665 & .235453 \\ 62 & 1 & 0 & 2.632517 & 4.629310 & .618904 \\ 63 & 1 & 0 & 4.181103 & 3.114175 & -.624676 \\ -\end{array}$

